\title{
En route to zero emissions for power and industry with amine-based post-combustion capture
}

\author{
David Danacia ${ }^{a}$, Mai Buib,c, Camille Petit ${ }^{a}$, Niall Mac Dowellb,c
}

a - Barrer Centre, Department of Chemical Engineering, Imperial College London, SW7 2AZ, UK

b - Centre for Environmental Policy, Imperial College London, SW7 1NE, UK

c - Centre for Process Systems Engineering, Imperial College London, SW7 2AZ, UK

\section{Number of pages: 44}

\section{List of figures:}

Figure S1 - The cost of capture as a function of amine lean loading for selected flue gas concentrations and capture rates at a flow rate of $500 \mathrm{~kg} \cdot \mathrm{s}^{-1}$.

Figure S2 - Specific reboiler duties and capture costs as a function of L/G ratio (lean loading) for a flue gas flow rate of $500 \mathrm{~kg} \cdot \mathrm{s}^{-1}$ and a capture rate of $90 \% \mathrm{~mol}$.

Figure S3 - Impact of accounting for additional fixed capital and operating \& maintenance costs on capture cost for a capture rate of $90 \%$. Capture cost as reported in the manuscript (A); capture cost including these additional costs (B); difference in cost as a multiple of the "excluding" cost, isolines for $2 \times$ and $3 \times$ are shown $(\mathrm{C})$.

Figure S4 - Effect of steam cost (columns) on capture cost at four different capture rates (rows), $70 \%$, $80 \%, 90 \%$, and $99 \%$.

Figure S5 - Effect of steam cost (columns) on total annualised cost at four different capture rates (rows), $70 \%, 80 \%, 90 \%$, and $99 \%$.

Figure S6 - Effect of steam cost (columns) on operating cost fraction at four different capture rates (rows), 70\%, 80\%, 90\%, and 99\%.

Figure S7 - Effect of reduced regeneration energy requirements on cost of capture as a function of gas composition and flow rate at four different capture rates, $70 \%, 80 \%, 90 \%$, and $99 \%$. 
Figure S8 - Percentage cost reduction relative to MEA due to reduced regeneration energy requirements as a function of gas composition and flow rate at four different capture rates, $70 \%, 80 \%, 90 \%$, and $99 \%$.

Figure S9 - Effect of reduced regeneration energy requirements on total annualized cost (A, C, E, G) and the operating cost fraction (B, D, F, H) at four different capture rates, $70 \%, 80 \%, 90 \%$, and $99 \%$. The colormap for the TAC plots (left column) uses a logarithmic scale.

Figure S10 - Figure 3 rescaled to emphasise marginal cost (solid lines) changes as a function of flow rate for a fixed capture rate of $90 \%$.

Figure S11 - Parity and residuals plots of the surrogate model fit.

Figure S12 - Comparison between VLE models for the CO2 - MEA system.

Figure S13 - Installed cost curves for a blower and compressor as a function of inlet volumetric flow rate.

Figure S14 - Comparison between $\mathrm{T}^{\text {sat }}$ data of water and output from surrogate function over the applicable pressure range.

Figure S15 - Comparison between Psat data of water and output from surrogate function over the applicable temperature range.

Figure S16 - Comparison between surrogate model and input data for the adiabatic index of the feed gas. Parity plot (left) and residuals plot (right).

Figure S17 - Wet acid gas heat capacity data points at selected pressures over temperature compared with the surrogate model predictions.

S38

Figure S18 - Comparison between surrogate model and input data for the wet acid gas heat capacity.

Parity plot (left) and residuals plot (right).

\section{List of tables:}

Table S1 - Capture costs in US\$.t $\mathrm{CO}_{2}^{-1}$ at different capture rates for the reference post-combustion capture applications, at reduced regeneration energy requirements.

Table S2 - Absolute error statistics of the surrogate model fit.

Table S3 - Percentage error statistics of the surrogate model fit.

Table S4 - Unit operation size limits. 


\section{S1. Table of Contents}

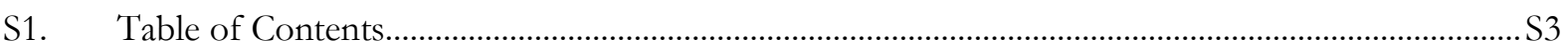

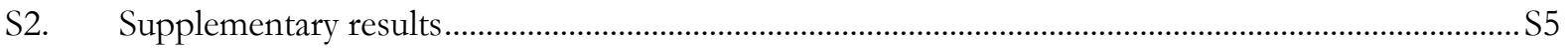

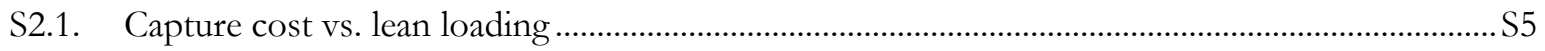

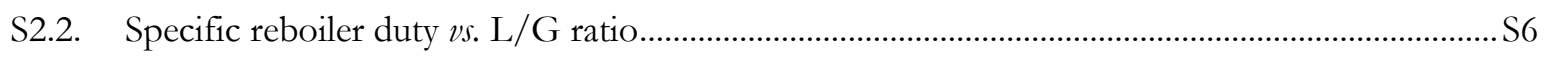

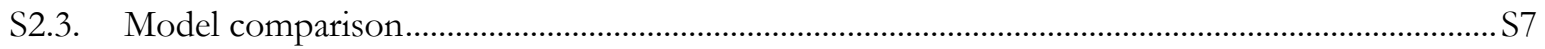

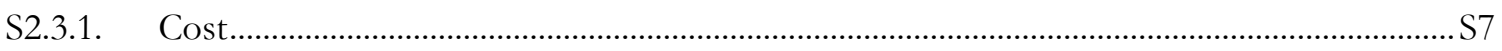

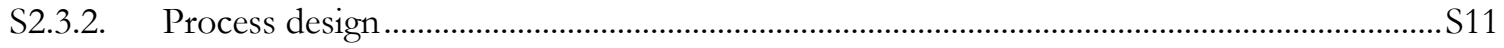

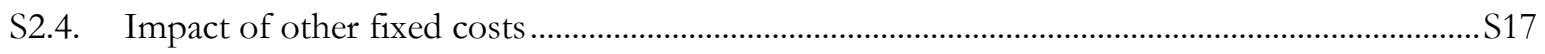

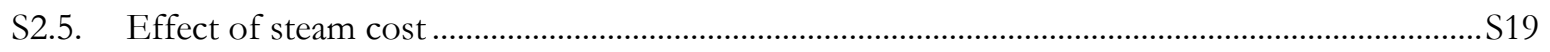

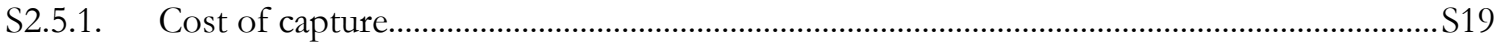

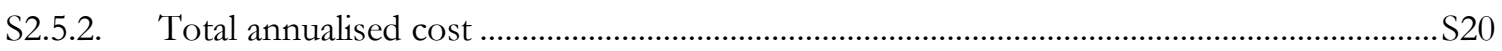

S2.5.3. Operating cost fraction.................................................................................................. 22

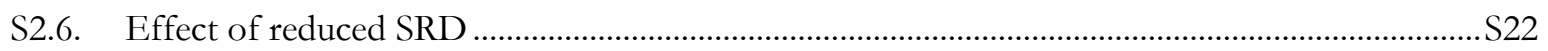

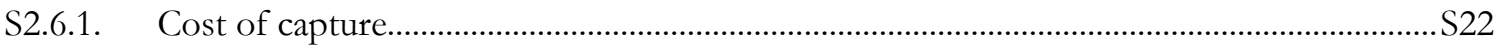

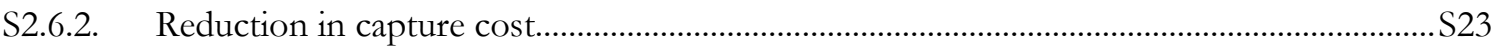

S2.6.3. Total annualised cost and operating cost fraction..................................................................... 24

S2.7. Marginal cost as a function of flow rate - rescaled.....................................................................S25

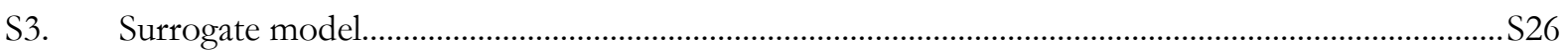

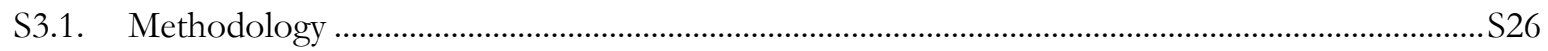

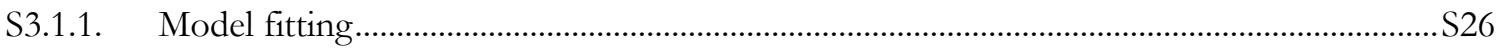

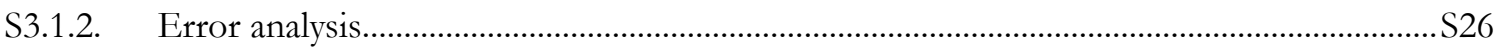

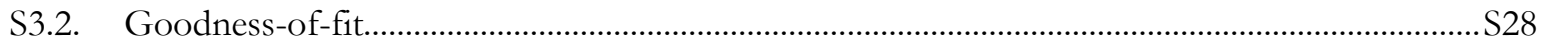

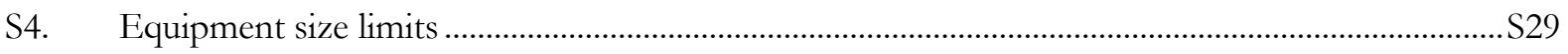

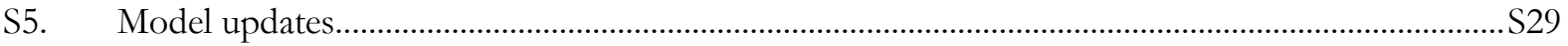

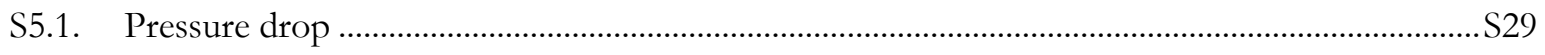

S5.2. Absorber rich amine outlet temperature ..................................................................................... 29

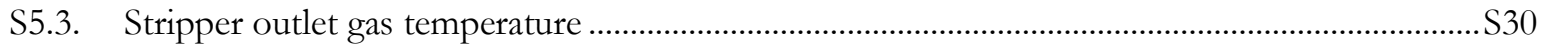

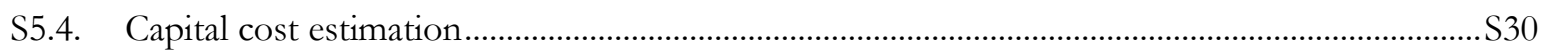




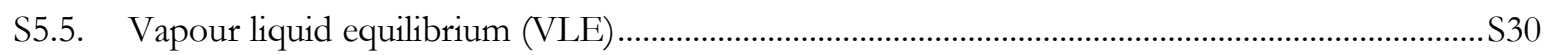

S5.5.1. Case 1 - T, P, yco2 are specified ………………………………………………………....... 31

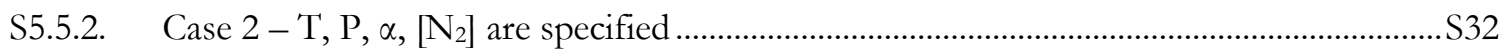

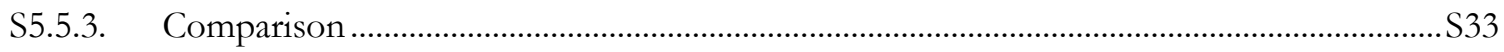

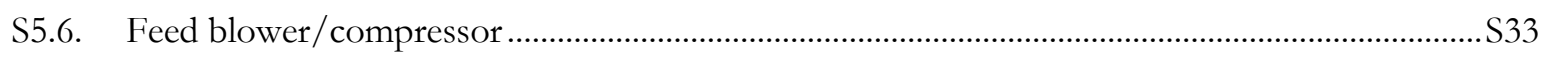

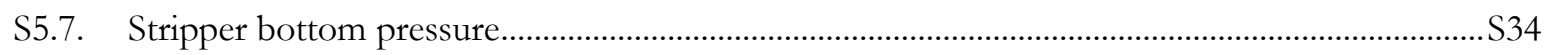

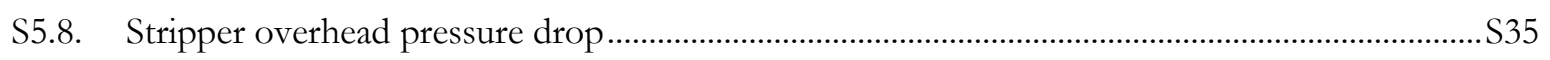

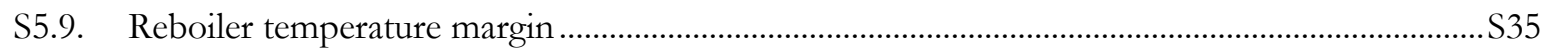

S5.10. Thermophysical properties of water …………........................................................................... 35

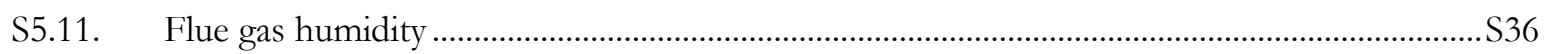

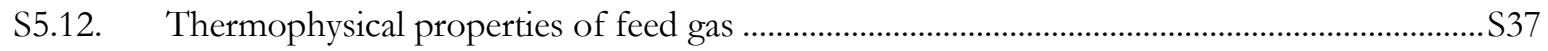

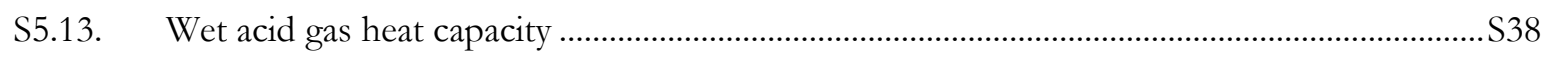

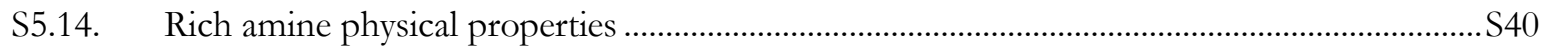

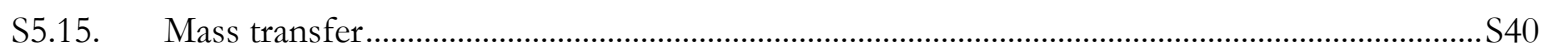

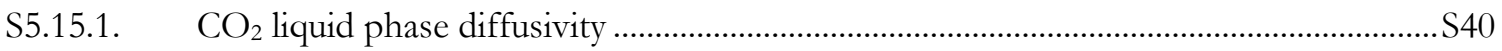

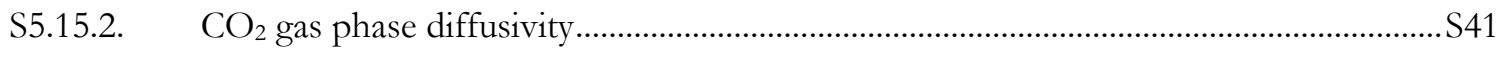

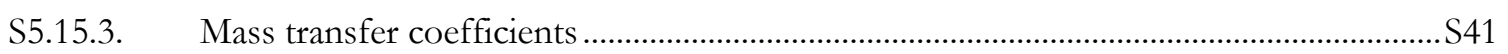

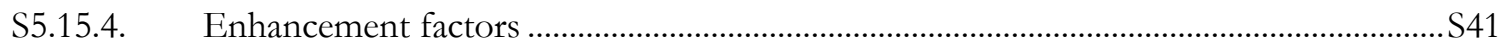

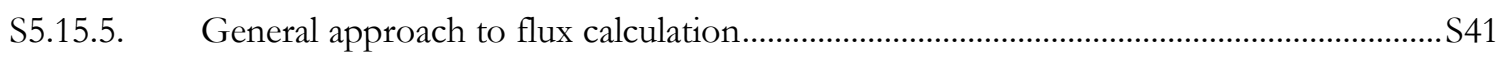

S5.16. Pump cost curves .......................................................................................................... 442

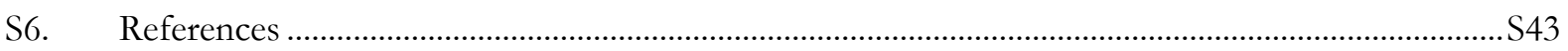




\section{S2. Supplementary results}

\section{S2.1. Capture cost vs. lean loading}

The effect of lean loading on capture cost was investigated for selected flue gas $\mathrm{CO}_{2}$ compositions. It was decided to opt for a fixed lean loading, if possible, in order to eliminate the variable of lean loading from the analysis.

For a flue gas flow rate of $500 \mathrm{~kg} \cdot \mathrm{s}^{-1}$, the cost of capture as a function of lean loading for selected flue gas compositions and capture rates is shown below.
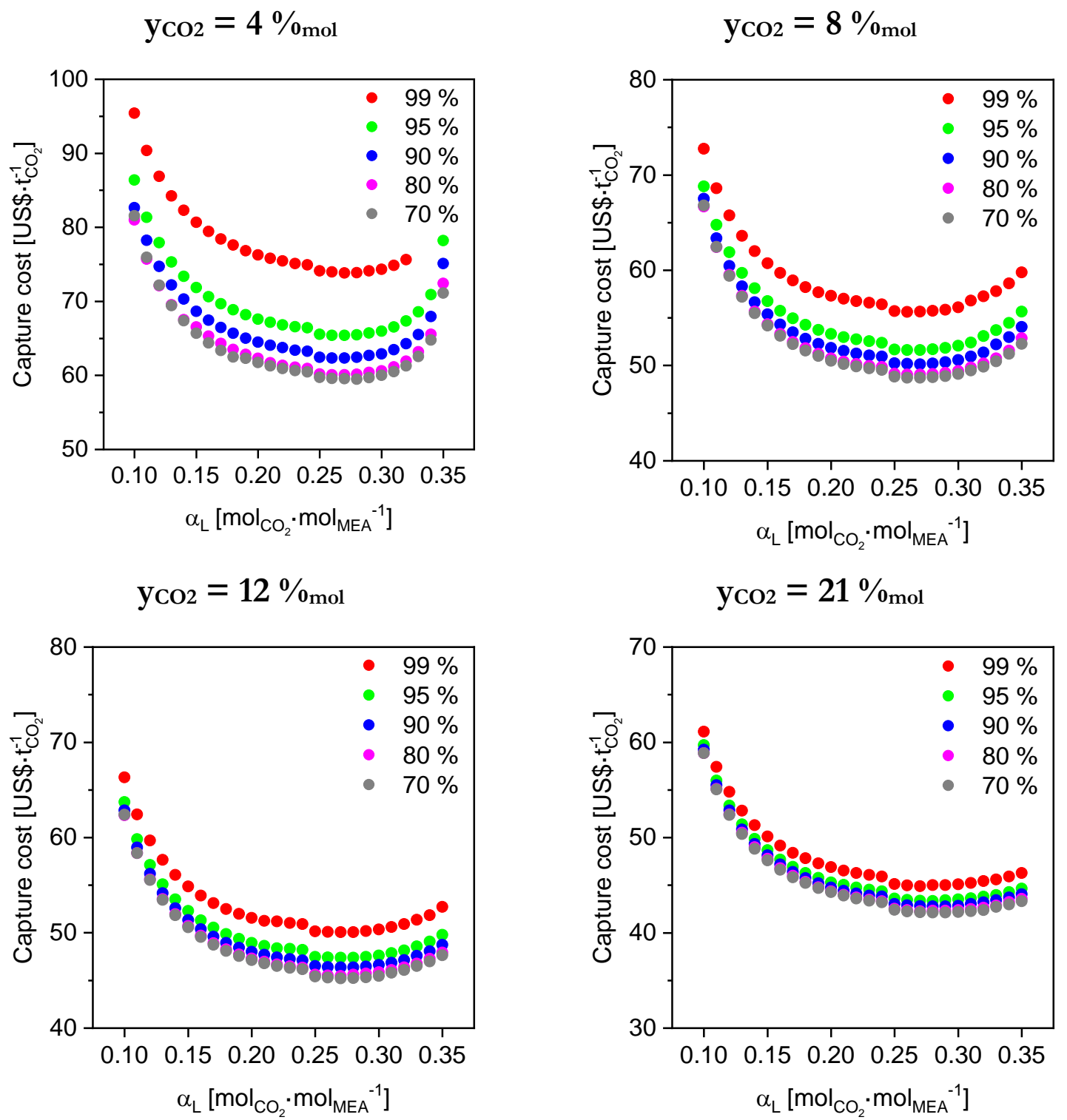

Figure $\mathbf{S} 1$ - The cost of capture as a function of amine lean loading for selected flue gas concentrations and capture rates at a flow rate of $500 \mathrm{~kg}^{-1} \mathrm{~s}^{-1}$. 


\section{S2.2. $\quad$ Specific reboiler duty vs. L/G ratio}

For completeness, the specific reboiler duties for flue gas compositions of 4 and $12 \%$ mol are shown below.
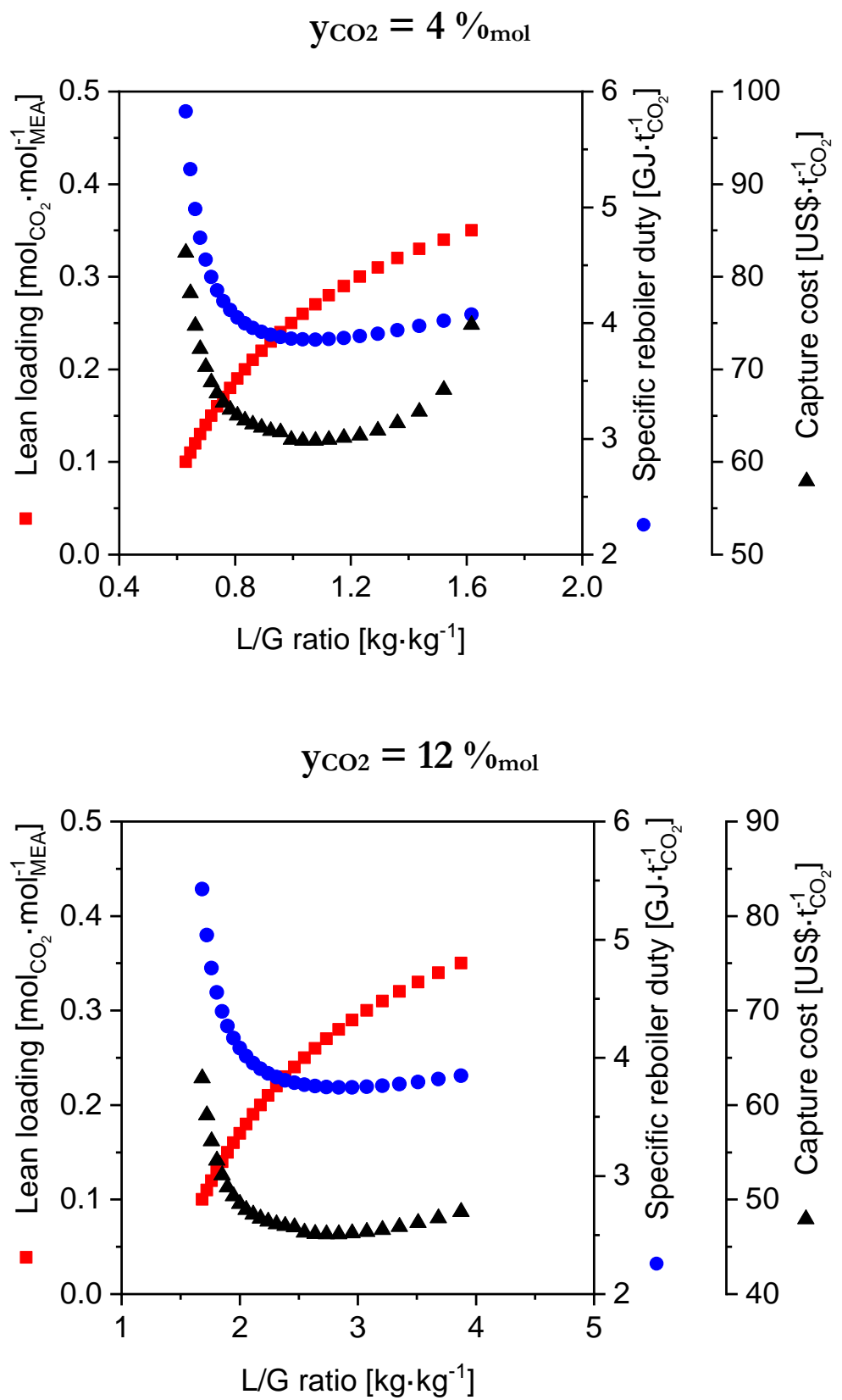

Figure S2 - Specific reboiler duties and capture costs as a function of $L / G$ ratio (lean loading) for a flue gas flow rate of $500 \mathrm{~kg} \cdot \mathrm{s}^{-1}$ and a capture rate of $90 \% \mathrm{~mol}$. 


\section{S2.3. Model comparison}

Below are comparisons of the output from the model used in this work, to existing publications. The comparisons on the basis of cost use the same parameters and assumptions described in the manuscript. The comparisons of the process design output match the process design and operating conditions described in the table; however, the remaining variables are the default values as described in the model description. Any inputs indicated as "assumed" are due to that information not being provided in the reference.

\section{S2.3.1. Cost}

Where required an exchange rate of $0.90 £: 1 €$, and $0.76 £: 1 \mathrm{US} \$$ was used. Costs are capture cost unless otherwise indicated. The 'costing differences' are a summary of the main differences in the reference work which would contribute disparity in the cost of capture.

\begin{tabular}{|c|c|c|}
\hline Reference & \multicolumn{2}{|c|}{ Garðarsdóttir et al. ${ }^{1}$} \\
\hline Capture rate $(\%)$ & \multicolumn{2}{|c|}{90} \\
\hline $\mathrm{yCO}$ & 0.30 & 0.13 \\
\hline Flow rate & $136.5 \mathrm{Nm}^{3} \cdot \mathrm{s}^{-1}$ & $133 \mathrm{Nm}^{3} \cdot \mathrm{s}^{-1}$ \\
\hline Cost $\left(\mathrm{US} \$ \cdot \mathrm{t}_{\mathrm{CO} 2}{ }^{-1}\right)$ & 59.7 & 73.2 \\
\hline This model & 41.7 & 46.9 \\
\hline Percentage difference [\%] & -30.2 & -35.9 \\
\hline Costing differences & $\begin{array}{l}\text { Conventional process lay } \\
\text { included, equipment cos } \\
\text { cost factors are based on } \\
\text { maintenance and labour }\end{array}$ & $\begin{array}{l}\text { oling and compression } \\
\text { mic Analyser, installed } \\
\text { ontingency included, }\end{array}$ \\
\hline
\end{tabular}

\begin{tabular}{|r|c|c|c|c|}
\hline Reference & \multicolumn{5}{|c|}{ Ho et al. } \\
\hline Capture rate (\%) & \multicolumn{5}{|c|}{90} \\
\hline yco2 & 0.13 & 0.20 & 0.09 & 0.22 \\
\hline Flow rate & $630 \mathrm{~kg} \cdot \mathrm{s}^{-1}$ & $393 \mathrm{~kg} \cdot \mathrm{s}^{-1}$ & $11 \mathrm{~kg} \cdot \mathrm{s}^{-1}$ & $70 \mathrm{~kg} \cdot \mathrm{s}^{-1}$ \\
\hline Cost (US\$.tco2-1) & 45.9 & 66.5 & 77.1 & 66.2 \\
\hline \multicolumn{5}{|c|}{ Reference costs are for $\mathrm{CO}_{2}$ avoided } \\
\hline This model & 46.0 & 43.4 & 65.6 & 44.1 \\
\hline Percentage difference [\%] & 0.218 & -34.7 & -14.9 & -33.3 \\
\hline Costing differences & $\begin{array}{l}\text { Conventional process layout, CO } 2 \text { compression included, flue gas } \\
\text { SCR \& FGD included, maintenance, labour, insurance, and taxes } \\
\text { included, some capital costs based on internal data. }\end{array}$ \\
\hline
\end{tabular}




\begin{tabular}{|c|c|}
\hline Reference & Mores et al. ${ }^{3}$ \\
\hline Capture rate $(\%)$ & 90 \\
\hline ycO2 & 0.04 (assumed) \\
\hline Flow rate & $\begin{array}{c}1100 \mathrm{~kg} \cdot \mathrm{s}^{-1} \\
\text { (assumed based on MWe output) }\end{array}$ \\
\hline Cost $\left(\mathrm{US} \$ \cdot \mathrm{t}_{\mathrm{CO}}{ }^{-1}\right)$ & 90.9 \\
\hline
\end{tabular}

\begin{tabular}{|r|c|}
\hline This model & 62.8 \\
\hline Percentage difference [\%] & -30.9 \\
\hline Costing differences & $\begin{array}{l}\text { Conventional process layout, } \mathrm{CO}_{2} \text { compression included, a range of } \\
\text { indirect capital costs included, labour, maintenance, taxes, insurance, } \\
\text { and administration costs included, equipment costs are scaled based } \\
\text { on a single size using an exponent of } 0.6 .\end{array}$ \\
\hline
\end{tabular}

\begin{tabular}{|c|c|}
\hline Reference & Nuchitprasittichai et al. ${ }^{4}$ \\
\hline Capture rate $(\%)$ & 90 \\
\hline ycO2 & 0.0244 \\
\hline Flow rate & $1365 \mathrm{kmol} \cdot \mathrm{hr}^{-1}$ \\
\hline Cost $\left(\mathrm{US} \$ \cdot \mathrm{t}_{\mathrm{CO} 2}{ }^{-1}\right)$ & 49.0 \\
\hline This model & 141.1 \\
\hline Percentage difference $[\%]$ & 188 \\
\hline Costing differences & $\begin{array}{l}\text { Conventional process layout, a range of indirect capital costs } \\
\text { included, labour, maintenance, taxes, insurance, and administration } \\
\text { costs included, numerical details of method to convert equipment } \\
\text { purchase cost to installed cost are not specified. }\end{array}$ \\
\hline
\end{tabular}

\begin{tabular}{|c|c|c|c|c|c|c|}
\hline Reference & \multicolumn{6}{|c|}{ Feron et al..$^{5}$} \\
\hline Capture rate $(\%)$ & 90 & 95 & 99 & 90 & 95 & 99 \\
\hline yсO2 & \multicolumn{3}{|c|}{0.134} & \multicolumn{3}{|c|}{0.042} \\
\hline Flow rate & \multicolumn{3}{|c|}{$826.8 \mathrm{~kg} \cdot \mathrm{s}^{-1}$} & \multicolumn{3}{|c|}{$1320 \mathrm{~kg} \cdot \mathrm{s}^{-1}$} \\
\hline Cost $\left(\mathrm{US} \$ \cdot \mathrm{t}_{\mathrm{CO}^{2}}{ }^{-1}\right)$ & 65.1 & 65.4 & 69.0 & 93.9 & 93.1 & 101.3 \\
\hline & \multicolumn{6}{|c|}{ Reference costs are for $\mathrm{CO}_{2}$ avoided } \\
\hline This model & 45.8 & 46.7 & 49.0 & 61.6 & 64.4 & 72.1 \\
\hline Percentage difference $[\%]$ & -29.6 & -28.6 & -29.0 & -34.4 & -30.8 & -28.8 \\
\hline Costing differences & \multicolumn{6}{|c|}{$\begin{array}{l}\text { Conventional process layout, a range of indirect capital costs } \\
\text { included, labour, maintenance, taxes, insurance, and administration } \\
\text { costs included, capital cost data from Aspen Capital Cost Estimator. }\end{array}$} \\
\hline
\end{tabular}




\begin{tabular}{|c|c|}
\hline Reference & Andersson et al. ${ }^{6}$ \\
\hline Capture rate $(\%)$ & 85 \\
\hline усO2 & 0.24 \\
\hline Flow rate & $150,000 \mathrm{Nm}^{3} \cdot \mathrm{hr}^{-1}$ \\
\hline \multirow[t]{2}{*}{ Cost $\left(\mathrm{US} \$ \cdot \mathrm{t}_{\mathrm{CO}^{-1}}\right)$} & 46.2 \\
\hline & Reference costs are for $\mathrm{CO}_{2}$ avoided \\
\hline This model & 43.8 \\
\hline Percentage difference $[\%]$ & -5.20 \\
\hline Costing differences & $\begin{array}{l}\text { Conventional process layout, } 15 \text { year economic life used, maintenance } \\
\text { costs included, costing based on } 400 \mathrm{ktpa} \text { plant and then scaled using } \\
\text { power law with exponent of } 0.6 \text {, a range of indirect capital costs } \\
\text { included, } 10 \% \text { contingency included on CapEx. }\end{array}$ \\
\hline
\end{tabular}

\begin{tabular}{|c|c|}
\hline Reference & 7 \\
\hline Capture rate $(\%)$ & 90 \\
\hline ycO2 & 0.133 \\
\hline Flow rate & $616 \mathrm{~kg} \cdot \mathrm{s}^{-1}$ \\
\hline \multirow[t]{2}{*}{ Cost $\left(\mathrm{US} \$ \cdot \mathrm{t}_{\mathrm{CO}^{-1}}{ }^{-1}\right)$} & 46.5 \\
\hline & Reference costs are for $\mathrm{CO}_{2}$ avoided \\
\hline This model & 45.8 \\
\hline Percentage difference $[\%]$ & -1.51 \\
\hline Costing differences & $\begin{array}{l}\text { Conventional process layout, } 7500 \mathrm{hrs} / \mathrm{yr} \text { plant uptime, maintenance } \\
\text { costs included, cost of } \mathrm{CO}_{2} \text { compression included, OSBL costs } \\
\text { included, range of indirect CapEx costs included, labour, } \\
\text { maintenance, taxes, insurance, and administration costs included. }\end{array}$ \\
\hline
\end{tabular}




\begin{tabular}{|c|c|c|c|c|}
\hline Reference & \multicolumn{4}{|c|}{ Agbonghae et al. ${ }^{8}$} \\
\hline Capture rate $(\%)$ & \multicolumn{4}{|c|}{90} \\
\hline yco2 & \multicolumn{2}{|c|}{0.0404} & 0.135 & 0.1238 \\
\hline Flow rate & $622.2 \mathrm{~kg} \cdot \mathrm{s}^{-1}$ & $725 \mathrm{~kg} \cdot \mathrm{s}^{-1}$ & $892.57 \mathrm{~kg} \cdot \mathrm{s}^{-1}$ & $932.42 \mathrm{~kg} \cdot \mathrm{s}^{-1}$ \\
\hline Cost $\left(\mathrm{US} \$ \cdot \mathrm{t}_{\mathrm{CO}^{2}}{ }^{-1}\right)$ & 65.6 & 67.7 & 58.8 & 58.1 \\
\hline This model & 63.0 & 62.8 & 45.7 & 46.4 \\
\hline Percentage difference [\%] & -3.96 & -7.24 & -22.3 & -20.1 \\
\hline Costing differences & $\begin{array}{l}\text { Conventional } \\
\text { Aspen Econo } \\
\text { costs specifie }\end{array}$ & $\begin{array}{l}\text { cess layout, } \\
\text { s Analyser b } \\
1 \text { process eq }\end{array}$ & $\begin{array}{l}\text { al and operatin } \\
\text { on default sett } \\
\text { ent constructe }\end{array}$ & $\begin{array}{l}\text { ts taken from } \\
\text { with utilities } \\
\text { n 316L SS. }\end{array}$ \\
\hline
\end{tabular}

\begin{tabular}{|c|c|c|c|c|c|c|c|}
\hline Reference & \multicolumn{7}{|c|}{ Hasan et al. ${ }^{9}$} \\
\hline Capture rate $(\%)$ & \multicolumn{7}{|c|}{90} \\
\hline $\mathrm{ycO}_{2}$ & 0.10 & 0.20 & 0.30 & 0.40 & 0.10 & 0.20 & 0.30 \\
\hline Flow rate $\left(\mathrm{kmol} \cdot \mathrm{s}^{-1}\right)$ & \multicolumn{4}{|c|}{0.1} & \multicolumn{3}{|c|}{1} \\
\hline Cost $\left(\mathrm{US} \$ \cdot \mathrm{t}_{\mathrm{CO}^{2}}{ }^{-1}\right)$ & 73.1 & 56.0 & 51.9 & 48.2 & 54.8 & 45.7 & 44.8 \\
\hline & \multicolumn{7}{|c|}{ Cost data digitised from Figure 18} \\
\hline This model & 91.5 & 64.4 & 55.7 & 51.5 & 54.6 & 46.7 & 43.3 \\
\hline Percentage difference $[\%]$ & 25.2 & 15.0 & 7.32 & 6.85 & -0.365 & 2.19 & -3.35 \\
\hline Capture rate $(\%)$ & \multicolumn{7}{|c|}{90} \\
\hline yco2 & \multicolumn{2}{|c|}{0.10} & \multicolumn{3}{|c|}{0.20} & \multicolumn{2}{|c|}{0.30} \\
\hline Flow rate $\left(\mathrm{kmol}^{\left.-\mathrm{s}^{-1}\right)}\right.$ & \multicolumn{7}{|c|}{10} \\
\hline Cost $\left(\mathrm{US} \$ \cdot \mathrm{t}_{\mathrm{CO}^{-1}}{ }^{-1}\right)$ & \multicolumn{2}{|c|}{42.0} & \multicolumn{3}{|c|}{35.9} & \multicolumn{2}{|c|}{35.0} \\
\hline & \multicolumn{7}{|c|}{ Cost data digitised from Figure 18} \\
\hline This model & \multicolumn{2}{|c|}{48.4} & \multicolumn{3}{|c|}{43.1} & \multicolumn{2}{|c|}{41.2} \\
\hline Percentage difference $[\%]$ & \multicolumn{2}{|c|}{15.2} & \multicolumn{3}{|c|}{20.1} & \multicolumn{2}{|c|}{17.7} \\
\hline Costing differences & \multicolumn{7}{|c|}{$\begin{array}{l}\text { Conventional process layout, costs of } \mathrm{CO}_{2} \text { compression included, } \\
\text { indirect capital costs, and maintenance costs included, higher capital } \\
\text { recovery factor. }\end{array}$} \\
\hline
\end{tabular}




\section{S2.3.2. Process design}

Reference: Bui et al. ${ }^{10}$

Type: Pilot plant data (TC Mongstad)

Flue gas temperature: $30^{\circ} \mathrm{C}$

Lean amine temperature: $37^{\circ} \mathrm{C}$

Absorber height $[\mathrm{m}] /$ area $\left[\mathrm{m}^{2}\right] /$ volume $\left[\mathrm{m}^{3}\right]:$ 24/7.1/170.4

\begin{tabular}{|c|c|c|c|c|c|c|}
\hline Run № & Initial & ICL1 & ICL2 & ICL3 & ICL4 & ICL5 \\
\hline \multicolumn{7}{|l|}{ Experimental data } \\
\hline $\mathrm{yCO} 2\left[\% \%_{\mathrm{vol}}\right]$ & 3.64 & 3.64 & 3.79 & 3.79 & 3.58 & 3.58 \\
\hline Flow rate $\left[\mathrm{kg} \cdot \mathrm{s}^{-1}\right]$ & 18.1 & 18.1 & 18.1 & 18.1 & 18.1 & 18.1 \\
\hline Capture rate $[\%]$ & 73.02 & 59.14 & 41.47 & 25.95 & 43.28 & 58.49 \\
\hline Lean loading $\left[\mathrm{mol}^{-} \cdot \mathrm{mol}^{-1}\right]$ & 0.1691 & 0.2814 & 0.3649 & 0.4375 & 0.3649 & 0.2814 \\
\hline Rich loading $\left[\mathrm{mol}^{\prime} \mathrm{mol}^{-1}\right]$ & 0.5176 & - & 0.5315 & - & - & 0.5217 \\
\hline $\mathrm{L} / \mathrm{G}\left[\mathrm{kg}^{\mathrm{k}} \mathrm{kg}^{-1}\right]$ & 0.699 & 0.895 & 0.902 & 0.915 & 0.903 & 0.892 \\
\hline SRD [GJ.t.tC2 $\left.2^{-1}\right]$ & 3.92 & 3.74 & 3.81 & 4.11 & 3.79 & 3.77 \\
\hline \multicolumn{7}{|l|}{ Model output } \\
\hline Absorber packed height [m] & 39.4 & 25.3 & 14.4 & 7.2 & 15.3 & 25.2 \\
\hline Absorber diameter $[\mathrm{m}]$ & 3.0 & 3.1 & 3.2 & 3.4 & 3.2 & 3.1 \\
\hline Absorber packed volume $\left[\mathrm{m}^{3}\right]$ & 279 & 191 & 116 & 65 & 123 & 190 \\
\hline Rich loading $\left[\mathrm{mol}^{-} \cdot \mathrm{mol}^{-1}\right]$ & 0.508 & 0.508 & 0.509 & 0.509 & 0.507 & 0.508 \\
\hline $\mathrm{L} / \mathrm{G}\left[\mathrm{kg}^{\prime} \mathrm{kg}^{-1}\right]$ & 0.57 & 0.69 & 0.79 & 1.00 & 0.79 & 0.67 \\
\hline $\mathrm{SRD}\left[\mathrm{GJ} \cdot \mathrm{tCO}^{-1}\right]$ & 4.27 & 3.85 & 4.13 & 5.49 & 4.11 & 3.85 \\
\hline \multicolumn{7}{|l|}{ Percentage deviation [\%] } \\
\hline Absorber packed volume & 63.4 & 12.1 & -32.0 & -61.6 & -27.8 & 11.6 \\
\hline Rich loading & -1.85 & - & -4.23 & - & - & -2.63 \\
\hline $\mathrm{L} / \mathrm{G}$ & -18.4 & -22.9 & -12.5 & 9.3 & -12.5 & -24.9 \\
\hline SRD & 8.93 & 2.94 & 8.40 & 33.6 & 8.44 & 2.12 \\
\hline
\end{tabular}

Continued... 


\begin{tabular}{|c|c|c|c|c|c|c|}
\hline Run No & ICL6 & ICL7 & ICL8 & ICL9 & ICL10 & ICL11 \\
\hline \multicolumn{7}{|l|}{ Experimental data } \\
\hline yCO2 $\left[\% \%_{\mathrm{vol}}\right]$ & 3.6 & 3.6 & 3.59 & 3.91 & 4.02 & 4.07 \\
\hline Flow rate $\left[\mathrm{kg} \cdot \mathrm{s}^{-1}\right]$ & 18.1 & 12.5 & 18.1 & 12.6 & - & 9.0 \\
\hline Capture rate $[\%]$ & 71.96 & 97.32 & 13.68 & 88.7 & 88.5 & 88.5 \\
\hline Lean loading $\left[\mathrm{mol}^{\prime} \cdot \mathrm{mol}^{-1}\right]$ & 0.2456 & 0.158 & 0.483 & 0.1556 & - & 0.1428 \\
\hline Rich loading $\left[\mathrm{mol}^{\prime} \cdot \mathrm{mol}^{-1}\right]$ & - & 0.5237 & 0.5261 & 0.5225 & - & 0.527 \\
\hline $\mathrm{L} / \mathrm{G}\left[\mathrm{kg}^{\mathrm{k}} \mathrm{kg}^{-1}\right]$ & 0.884 & 0.877 & 0.922 & 0.872 & - & 0.872 \\
\hline $\mathrm{SRD}\left[\mathrm{GJ} \cdot \mathrm{t}_{\mathrm{CO}_{2}}{ }^{-1}\right]$ & 3.9 & 4.2 & 12.3 & 4.1 & 4.27 & 4.28 \\
\hline \multicolumn{7}{|l|}{ Model output } \\
\hline Absorber packed height [m] & 34.7 & 94.5 & 2.9 & 57.9 & & 57.2 \\
\hline Absorber diameter $[\mathrm{m}]$ & 3.1 & 2.6 & 3.7 & 2.6 & & 2.2 \\
\hline Absorber packed volume $\left[\mathrm{m}^{3}\right]$ & 262 & 502 & 31 & 307 & & 217 \\
\hline Rich loading $\left[\mathrm{mol} \cdot \mathrm{mol}^{-1}\right]$ & 0.508 & 0.509 & 0.507 & 0.511 & - & 0.512 \\
\hline $\mathrm{L} / \mathrm{G}\left[\mathrm{kg}^{2} \mathrm{~kg}^{-1}\right]$ & 0.71 & 0.72 & 1.47 & 0.70 & - & 0.7 \\
\hline $\mathrm{SRD}\left[\mathrm{GJ} \cdot \mathrm{t}_{\mathrm{CO}{ }^{-1}}\right]$ & 3.88 & 4.36 & 11.61 & 4.38 & - & 4.56 \\
\hline \multicolumn{7}{|l|}{ Percentage deviation [\%] } \\
\hline Absorber packed volume & 53.7 & 194.4 & -81.7 & 80.4 & & 27.6 \\
\hline Rich loading & - & -2.81 & -3.63 & -2.20 & - & -2.85 \\
\hline $\mathrm{L} / \mathrm{G}$ & -19.7 & -17.9 & 59.5 & -19.7 & - & -19.7 \\
\hline SRD & -0.513 & 3.81 & -5.61 & 6.83 & - & 6.54 \\
\hline
\end{tabular}

\begin{tabular}{|c|c|c|}
\hline Run № & ICL12 & ICL13 \\
\hline \multicolumn{3}{|l|}{ Experimental data } \\
\hline $\mathrm{yCO} 2\left[\% \%_{\mathrm{vol}}\right]$ & 4.07 & 4.09 \\
\hline Flow rate $\left[\mathrm{kg} \cdot \mathrm{s}^{-1}\right]$ & 10.8 & 12.5 \\
\hline Capture rate $[\%]$ & 88.51 & 86.91 \\
\hline Lean loading $\left[\mathrm{mol}^{-} \cdot \mathrm{mol}^{-1}\right]$ & 0.1513 & 0.1596 \\
\hline Rich loading $\left[\mathrm{mol}^{\prime} \cdot \mathrm{mol}^{-1}\right]$ & 0.5285 & 0.5321 \\
\hline $\mathrm{L} / \mathrm{G}\left[\mathrm{kg}^{\mathrm{k}} \cdot \mathrm{kg}^{-1}\right]$ & 0.877 & 0.880 \\
\hline $\mathrm{SRD}\left[\mathrm{GJ} \cdot \mathrm{t}_{\mathrm{CO} 2^{-1}}\right]$ & 4.2 & 4.1 \\
\hline \multicolumn{3}{|l|}{ Model output } \\
\hline Absorber packed height [m] & 56.7 & 53.2 \\
\hline Absorber diameter $[\mathrm{m}]$ & 2.4 & 2.6 \\
\hline Absorber packed volume $\left[\mathrm{m}^{3}\right]$ & 257 & 282 \\
\hline Rich loading $\left[\mathrm{mol}^{\prime} \cdot \mathrm{mol}^{-1}\right]$ & 0.512 & 0.512 \\
\hline $\mathrm{L} / \mathrm{G}\left[\mathrm{kg}^{\mathrm{k}} \mathrm{kg}^{-1}\right]$ & 0.70 & 0.72 \\
\hline $\mathrm{SRD}\left[\mathrm{GJ} \cdot \mathrm{t}_{\mathrm{CO}_{2}}{ }^{-1}\right]$ & 4.56 & 4.43 \\
\hline \multicolumn{3}{|l|}{ Percentage deviation [\%] } \\
\hline Absorber packed volume & 50.5 & 65.8 \\
\hline Rich loading & -2.85 & -3.12 \\
\hline $\mathrm{L} / \mathrm{G}$ & -19.7 & -17.9 \\
\hline SRD & 6.54 & 5.48 \\
\hline
\end{tabular}


Reference: Faramarzi et al. ${ }^{11}$

Type: Pilot plant data (TC Mongstad)

Flue gas temperature: $29.4{ }^{\circ} \mathrm{C}$

Lean amine temperature: $37^{\circ} \mathrm{C}$

Absorber height $[\mathrm{m}] /$ area $\left[\mathrm{m}^{2}\right] /$ volume $\left[\mathrm{m}^{3}\right]: 24 / 7.1 / 170.4$

\begin{tabular}{|c|c|}
\hline Experimental data & \\
\hline yCO2 $\left[\%{ }_{\mathrm{vol}}\right]$ & 3.56 \\
\hline Flow rate $\left[\mathrm{kg} \cdot \mathrm{s}^{-1}\right]$ & 17.8 \\
\hline Capture rate $[\%]$ & 83.4 \\
\hline Lean loading $\left[\mathrm{mol}^{\prime} \cdot \mathrm{mol}^{-1}\right]$ & 0.20 \\
\hline Rich loading $\left[\mathrm{mol} \cdot \mathrm{mol}^{-1}\right]$ & 0.48 \\
\hline $\mathrm{L} / \mathrm{G}\left[\mathrm{kg}^{\mathrm{kg}} \mathrm{kg}^{-1}\right]$ & 0.90 \\
\hline $\mathrm{SRD}\left[\mathrm{GJ} \cdot \mathrm{tCO}{ }^{-1}\right]$ & 3.62 \\
\hline Absorber packed volume $\left[\mathrm{m}^{3}\right]$ & 107.4 \\
\hline \multicolumn{2}{|l|}{ Model output } \\
\hline Rich loading $\left[\mathrm{mol} \cdot \mathrm{mol}^{-1}\right]$ & 0.508 \\
\hline $\mathrm{L} / \mathrm{G}\left[\mathrm{kg}^{\mathrm{k}} \cdot \mathrm{kg}^{-1}\right]$ & 0.70 \\
\hline $\mathrm{SRD}\left[\mathrm{GJ} \cdot \mathrm{tCO}^{-1}{ }^{-1}\right]$ & 4.03 \\
\hline Absorber packed height [m] & 48.9 \\
\hline Absorber diameter $[\mathrm{m}]$ & 3.1 \\
\hline Absorber packed volume $\left[\mathrm{m}^{3}\right]$ & 369 \\
\hline \multicolumn{2}{|l|}{ Percentage deviation $[\%]$} \\
\hline Rich loading & 5.83 \\
\hline $\mathrm{L} / \mathrm{G}$ & -27.6 \\
\hline SRD & 11.3 \\
\hline Absorber packed volume & 117 \\
\hline
\end{tabular}


Please refer to footnotes following the tables for details of references, model type, and any footnotes.

\begin{tabular}{|c|c|c|c|c|c|c|c|c|c|c|c|c|}
\hline \multicolumn{13}{|l|}{ Reference data } \\
\hline Reference & \multicolumn{2}{|c|}{ Agbonghae et al. ${ }^{8}$} & \multicolumn{2}{|c|}{ Alhajaj et al. ${ }^{12}$} & \multicolumn{2}{|c|}{ Canepa et al. ${ }^{13}$} & \multicolumn{4}{|c|}{ Feron et al. ${ }^{5}$} & \multicolumn{2}{|c|}{ Mores et al. ${ }^{3}$} \\
\hline Type & \multicolumn{2}{|c|}{ DAP } & \multicolumn{2}{|c|}{ SGP } & \multicolumn{2}{|c|}{ DAP } & \multicolumn{4}{|c|}{ DPT } & \multicolumn{2}{|c|}{ DGA } \\
\hline Flue gas pressure $\left[\mathrm{bar}_{\mathrm{a}}\right]$ & \multicolumn{2}{|c|}{$1.2^{\mathrm{T}}$} & \multicolumn{2}{|c|}{$1.01 \mathrm{U}$} & \multicolumn{2}{|c|}{$1.05^{\mathrm{U}}$} & \multicolumn{4}{|c|}{$1.05^{\mathrm{T}}$} & \multicolumn{2}{|c|}{$1.01 \mathrm{U}, \mathrm{V}$} \\
\hline Flue gas temperature $\left[{ }^{\circ} \mathrm{C}\right]$ & \multicolumn{2}{|c|}{40} & \multicolumn{2}{|c|}{50} & \multicolumn{2}{|c|}{40} & \multicolumn{4}{|c|}{$32 \mathrm{x}$} & \multicolumn{2}{|c|}{40} \\
\hline $\mathrm{yCO2}[\% \mathrm{~mol}]$ & \multicolumn{2}{|c|}{0.0404} & & & & & & & & & & \\
\hline Flow rate $\left[{\left.\mathrm{kg} \cdot \mathrm{s}^{-1}\right]}^{-1}\right.$ & & & & & & & & & & & & \\
\hline Capture rate $[\%]$ & & & & & & & & & & & & \\
\hline Amine lean loading & & & & & & & & & & & & \\
\hline Lean amine temperature $\left[{ }^{\circ} \mathrm{C}\right]$ & & & & & & & & & & & & \\
\hline Column flooding [\%] & & & & & & & & & & & & \\
\hline $\mathrm{L} / \mathrm{G}\left[\mathrm{kg}^{\mathrm{kg}}{ }^{-1}\right]$ & & & & & & & & & & & & \\
\hline Absorber & & & & & & & & & & & & \\
\hline Number & & & & & & & & & & & & \\
\hline Diameter $[\mathrm{m}]$ & & & & & & & & & & & & \\
\hline Height $[\mathrm{m}]$ & & & & & & & & & & & & \\
\hline Volume $\left[\mathrm{m}^{3}\right]$ & & & & & & & & & & & & \\
\hline Stripper & & & & & & & & & & & & \\
\hline Number & & & & & & & & & & & & \\
\hline Diameter $[\mathrm{m}]$ & & & & & & & & & & & & \\
\hline Height $[\mathrm{m}]$ & & & & & & & & & & & & \\
\hline Volume $\left[\mathrm{m}^{3}\right]$ & & & & & & & & & & & & \\
\hline SRD [GJ·t $\left.\mathrm{CO}^{-1}\right]$ & & & & & & & & & & & & \\
\hline \begin{tabular}{l|l} 
This model & Deviation [\%] \\
\end{tabular} & & & & & & & & & & & & \\
\hline $\mathrm{L} / \mathrm{G}\left[\mathrm{kg}^{\left.\cdot \mathrm{kg}^{-1}\right]}\right.$ & 0.88 & -8.3 & 1.54 & - & 0.86 & - & 0.87 & 3.6 & 0.97 & -9.35 & 0.82 & - \\
\hline Absorber & & & & & & & & & & & & \\
\hline Number & 2 & 0 & 1 & 0 & 2 & -33 & 3 & 200 & 3 & 200 & 2 & 100 \\
\hline Diameter $[\mathrm{m}]$ & 12.4 & 4 & 15.4 & 6 & 14.8 & 44 & 15.1 & 47 & 15.3 & 44.3 & 15.9 & 45 \\
\hline Height [m] & 52 & 173 & 35 & 19 & 53.2 & 113 & 57.2 & 186 & 134.4 & 572 & 60.2 & 131 \\
\hline Volume $\left[\mathrm{m}^{3}\right]$ & 12559 & 195 & 6519 & 34 & 18304 & 193 & 30730 & 1744 & 74130 & 4100 & 23906 & 866 \\
\hline Stripper & & & & & & & & & & & & \\
\hline Number & 1 & 0 & 1 & 0 & 1 & 0 & 1 & 0 & 1 & 0 & 1 & 0 \\
\hline Diameter $[\mathrm{m}]$ & 8 & 18 & 7.7 & -4 & 9.3 & 26 & 11.6 & 190 & 12.2 & 160 & 10.2 & 203 \\
\hline Height [m] & 10.8 & -62 & 41.4 & 137 & 10.4 & -32 & 9.6 & -4 & 9.7 & -3.00 & 6.9 & 9 \\
\hline Volume [m³] & 543 & -46 & 1928 & 119 & 706 & 10 & 1015 & 707 & 1134 & 555 & 564 & 900 \\
\hline $\mathrm{SRD}\left[\mathrm{GJ} \cdot \mathrm{t}_{\mathrm{CO}^{-1}}{ }^{-1}\right.$ & 4.00 & 1.01 & 4.06 & -9.46 & 4.02 & -1.95 & 4.00 & 12.4 & 4.00 & -10.3 & 4.49 & - \\
\hline
\end{tabular}




\begin{tabular}{|c|c|c|c|c|c|c|}
\hline \multicolumn{7}{|l|}{ Reference data } \\
\hline Reference & \multicolumn{2}{|c|}{ Agbonghae et al. ${ }^{8}$} & \multicolumn{2}{|c|}{ Garðarsdóttir et al. ${ }^{1}$} & \multicolumn{2}{|c|}{ Jiang et al..$^{14}$} \\
\hline Type & \multicolumn{2}{|c|}{ DAP } & \multicolumn{2}{|c|}{ DAP } & \multicolumn{2}{|c|}{ DAP } \\
\hline Flue gas pressure [bar $\left.{ }_{\mathrm{a}}\right]$ & \multicolumn{2}{|c|}{$1.2^{\mathrm{T}}$} & \multicolumn{2}{|c|}{$1 \mathrm{U}$} & \multicolumn{2}{|c|}{$1.01 \mathrm{U}, \mathrm{V}$} \\
\hline Flue gas temperature $\left[{ }^{\circ} \mathrm{C}\right]$ & \multicolumn{2}{|c|}{40} & \multicolumn{2}{|c|}{40} & \multicolumn{2}{|c|}{$35^{\mathrm{V}}$} \\
\hline $\mathrm{yCO} 2[\% \mathrm{~mol}]$ & \multicolumn{2}{|c|}{0.1238} & \multicolumn{2}{|c|}{0.13} & \multicolumn{2}{|c|}{0.1288} \\
\hline Flow rate $\left[\mathrm{kg} \cdot \mathrm{s}^{-1}\right]$ & \multicolumn{2}{|c|}{892.6} & \multicolumn{2}{|c|}{$133 \mathrm{Nm}^{3} / \mathrm{s}$} & \multicolumn{2}{|c|}{285.4} \\
\hline Capture rate $[\%]$ & \multicolumn{2}{|c|}{90} & \multicolumn{2}{|c|}{90} & \multicolumn{2}{|c|}{90} \\
\hline Amine lean loading & \multicolumn{2}{|c|}{0.20} & \multicolumn{2}{|c|}{0.26} & \multicolumn{2}{|c|}{0.25} \\
\hline Lean amine temperature $\left[{ }^{\circ} \mathrm{C}\right]$ & \multicolumn{2}{|c|}{40} & \multicolumn{2}{|c|}{40} & \multicolumn{2}{|c|}{40} \\
\hline Column flooding $[\%]$ & & & & & & \\
\hline $\mathrm{L} / \mathrm{G}\left[\mathrm{kg}^{\mathrm{k}} \cdot \mathrm{kg}^{-1}\right]$ & & & & & & \\
\hline Absorber & & & & & & \\
\hline Number & & & & & & \\
\hline Diameter [m] & & & & & & \\
\hline Height $[\mathrm{m}]$ & & & & & & \\
\hline Volume $\left[\mathrm{m}^{3}\right]$ & & & & & & \\
\hline Stripper & & & & & & \\
\hline Number & & & & & & \\
\hline Diameter $[\mathrm{m}]$ & & & & & & \\
\hline Height $[\mathrm{m}]$ & & & & & & \\
\hline Volume $\left[\mathrm{m}^{3}\right]$ & & & & & & \\
\hline $\mathrm{SRD}\left[\mathrm{G} J \cdot \mathrm{t}_{\mathrm{CO} 2^{-1}}\right]$ & & & & & & \\
\hline \begin{tabular}{l|l} 
This model & Deviation [\%] \\
\end{tabular} & & & & & & \\
\hline $\mathrm{L} / \mathrm{G}\left[\mathrm{kg}^{2} \mathrm{~kg}^{-1}\right]$ & 2.31 & -21.2 & 3.01 & -11.5 & 2.78 & -10.0 \\
\hline Absorber & & & & & & \\
\hline Number & 3 & 50 & 1 & 0 & 2 & 100 \\
\hline Diameter [m] & 15.5 & -7.02 & 12.2 & - & 11.5 & -4.17 \\
\hline Height $[\mathrm{m}]$ & 29.6 & 28.5 & 28.3 & 13.2 & 29.6 & 48.0 \\
\hline Volume $\left[\mathrm{m}^{3}\right]$ & 16756 & 66.6 & 3308 & - & 6149 & 172 \\
\hline Stripper & & & & & & \\
\hline Number & 1 & 0 & 1 & 0 & 1 & 0 \\
\hline Diameter $[\mathrm{m}]$ & 15.7 & 10.2 & 7.2 & - & 9.5 & 35.7 \\
\hline Height $[\mathrm{m}]$ & 9.9 & -61.4 & 14.1 & 17.5 & 12.3 & 105 \\
\hline Volume $\left[\mathrm{m}^{3}\right]$ & 1917 & -53.1 & 574 & - & 872 & 278 \\
\hline $\mathrm{SRD}\left[\mathrm{GJ} \cdot \mathrm{t}_{\mathrm{CO} 2^{-1}}\right]$ & 3.92 & 6.23 & 3.80 & 0 & 3.79 & 7.67 \\
\hline
\end{tabular}




\section{Table footnotes}

Types: DAP - detailed Aspen Plus model, SGP - simplified gPROMS model, DPT - detailed ProTreat model, DGA - detailed GAMS model

Footnotes: $\mathrm{T}$ - absorber inlet, $\mathrm{U}$ - absorber outlet, $\mathrm{V}$ - assumption, $\mathrm{W}$ - derived from other provided information, $\mathrm{X}$ - digitised from figure 


\section{S2.4. Impact of other fixed costs}

In order to demonstrate the potential effect of including other fixed costs, a comparison is presented below for the $90 \%$ capture rate case. Reference values for the additional fixed costs, and operating and maintenance costs are taken from Jiang et al. ${ }^{14}$ The additional costs accounted for are listed below.

\begin{tabular}{|c|c|}
\hline Capital costs & \\
\hline Bare erected cost (BEC) & CapEx as described in manuscript \\
\hline Engineering, procurement, and construction (EPC) & $10 \%$ of $\mathrm{BEC}$ \\
\hline Process contingency (PROC) & $15.9 \%$ of $(\mathrm{BEC}+\mathrm{EPC})$ \\
\hline Project contingency (PRJC) & $20.7 \%$ of $(\mathrm{BEC}+\mathrm{EPC}+\mathrm{PROC})$ \\
\hline Total plant cost (TPC) & Sum of above \\
\hline Modifications & $2 \%$ of TPC \\
\hline Other costs & $15 \%$ of $\mathrm{TPC}$ \\
\hline Total overnight costs (TOC) & Sum of above \\
\hline As-spent cost multiplier & 1.14 \\
\hline Total capital cost (TCPX) & $(\mathrm{TPC}+\mathrm{TOC}) \times 1.14$ \\
\hline \multicolumn{2}{|l|}{ Operating \& Maintenance costs } \\
\hline Maintenance costs & $1.5 \%$ of $\mathrm{TPC} / \mathrm{yr}$ \\
\hline Operator labour rate (LR) & $\$ 45 / \mathrm{hr}$ \\
\hline Number of operators (NO) & 16 \\
\hline Operator shift/fraction (OF) & 0.3 \\
\hline Operating hours $(\mathrm{OH})$ & $8760 \mathrm{hrs} / \mathrm{yr}$ \\
\hline Total operating labour (TOL) & $(\mathrm{LR} \times \mathrm{NO} \times \mathrm{OR} \times \mathrm{OH})$ \\
\hline Administration \& support labour (ASL) & $30 \%$ of TOL \\
\hline Insurance \& taxes (IAT) & $2 \%$ of TPC \\
\hline Fixed $O \& M$ costs (FOM) & TOL + ASL + IAT \\
\hline Variable $O \& M$ costs (VOC) & OpEx as described in manuscript \\
\hline Total O\&M costs (TOMC) & FOM + VOC \\
\hline
\end{tabular}

The capture cost was calculated using the same procedure described in the manuscript, however, the CapEx is replaced with the TCPX and the OpEx is replaced with the TOMC. 

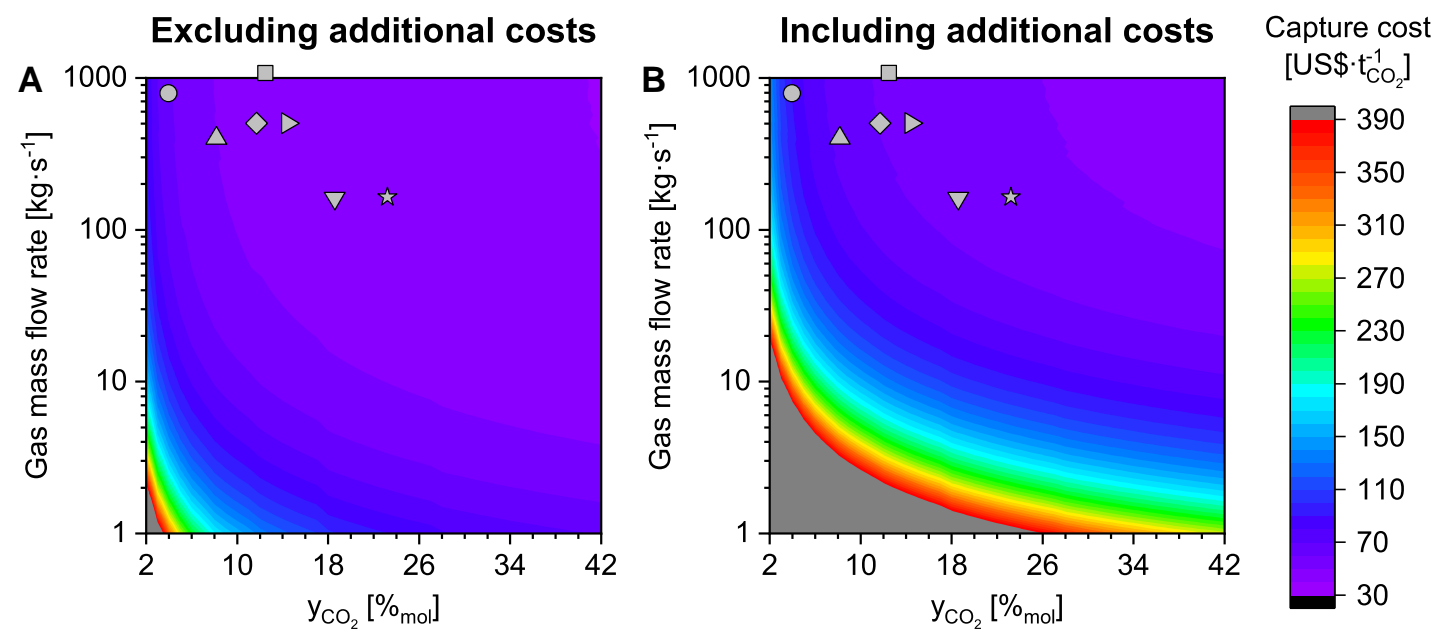

Cost difference (multiple) Cost multiple $[\times]$

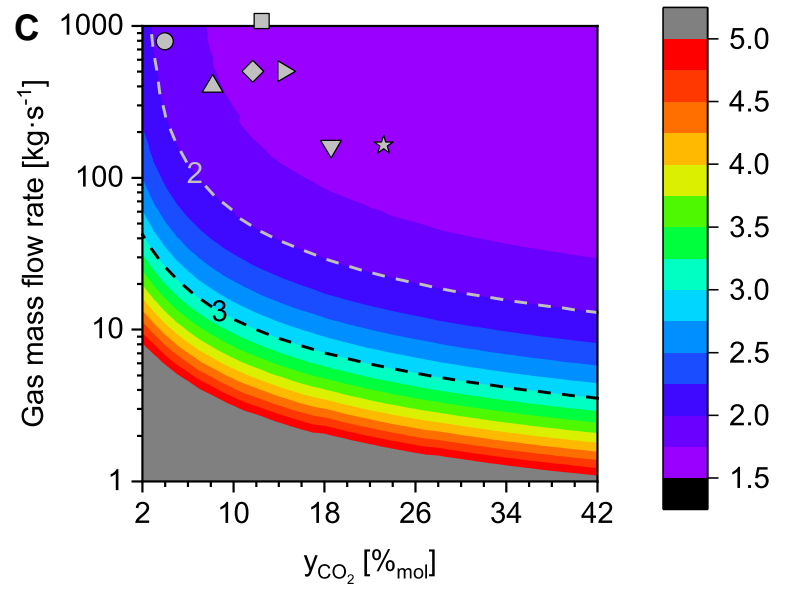

○ 500 MW NGCC $\triangle 500$ MW NGCC 50 \% EGR $\diamond 500$ MW high-rank PC $\square \quad 500$ MW low-rank PC $\triangleright 500 \mathrm{MW}$ biomass $\nabla 1 \mathrm{MMtpa}$ cement is 1 MMtpa steel

Figure S3 - Impact of accounting for additional fixed capital and operating \& maintenance costs on capture cost for a capture rate of $90 \%$. Capture cost as reported in the manuscript (A); capture cost including these additional costs (B); difference in cost as a multiple of the "excluding" capture cost, isolines for $2 \times$ and $3 \times$ are shown $(C)$. 


\section{S2.5. Effect of steam cost}

\section{S2.5.1. Cost of capture}
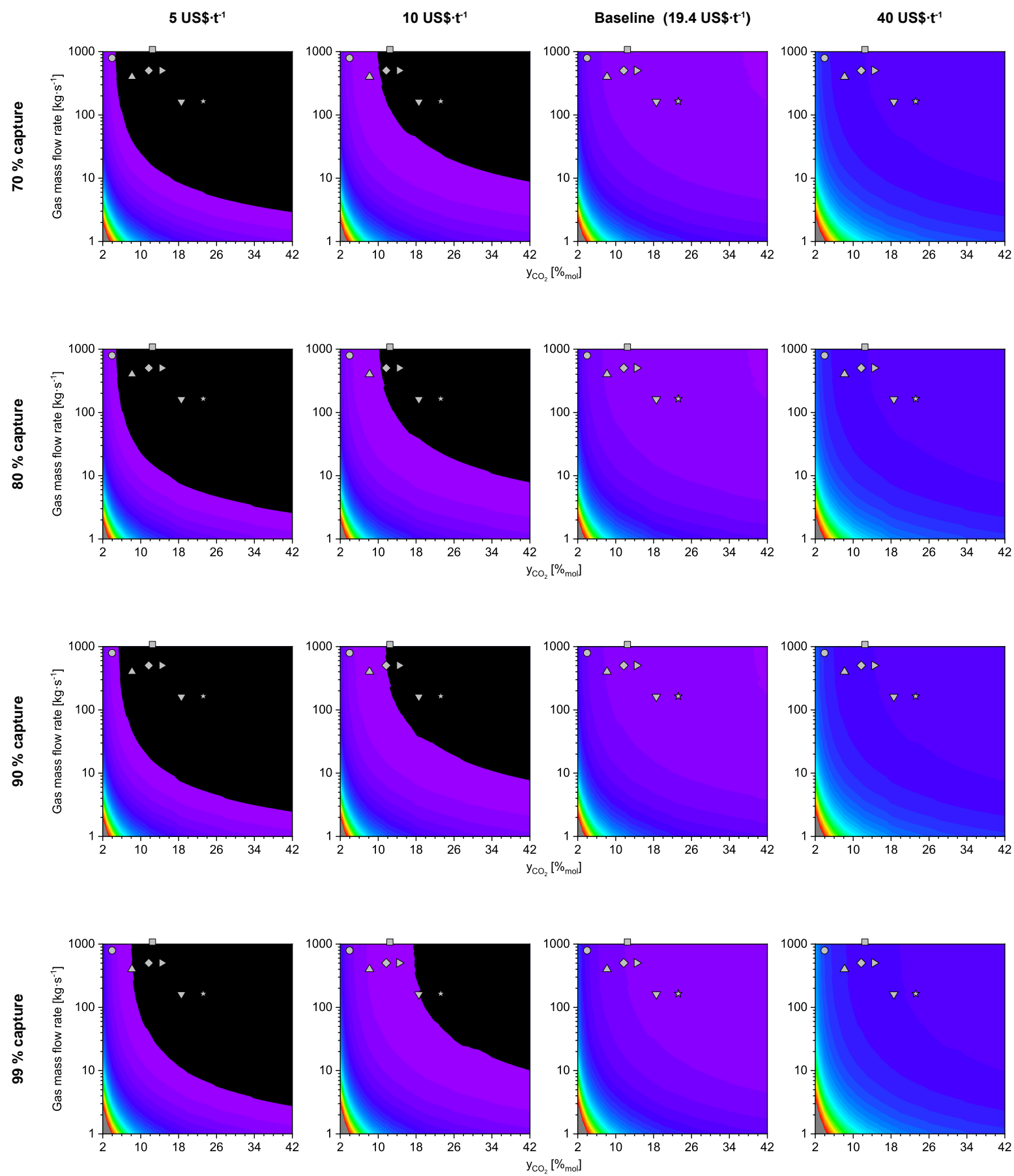

- 500 MW NGCC $\triangle 500$ MW NGCC $50 \%$ EGR $\diamond 500$ MW high-rank PC $\square 500$ MW low-rank PC $\triangleright 500 \mathrm{MW}$ biomass $\nabla 1 \mathrm{MMtpa}$ cement $म$ म $1 \mathrm{MMtpa}$ steel

Figure S4 - Effect of steam cost (columns) on capture cost at four different capture rates (rows), $70 \%$, $80 \%, 90 \%$, and $99 \%$. 
S2.5.2. Total annualised cost
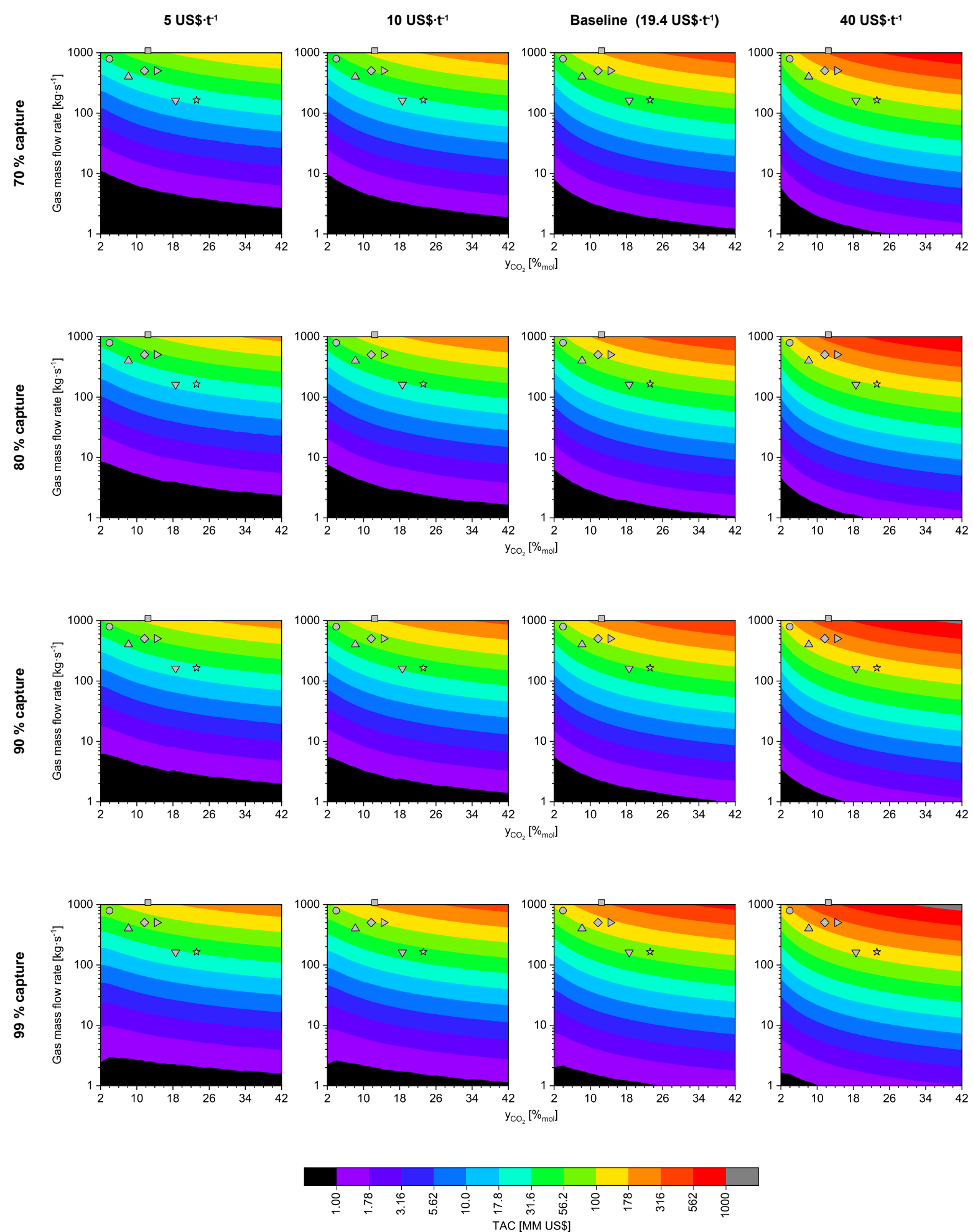

○ 500 MW NGCC $\triangle 500$ MW NGCC $50 \%$ EGR $\diamond 500$ MW high-rank PC $\square 500$ MW low-rank PC $\triangleright 500 \mathrm{MW}$ biomass $\nabla 1 \mathrm{MMtpa}$ cement $\Rightarrow 1 \mathrm{MMtpa}$ steel

Figure S5 - Effect of steam cost (columns) on total annualised cost at four different capture rates (rows), $70 \%, 80 \%, 90 \%$, and $99 \%$. 
S2.5.3. Operating cost fraction
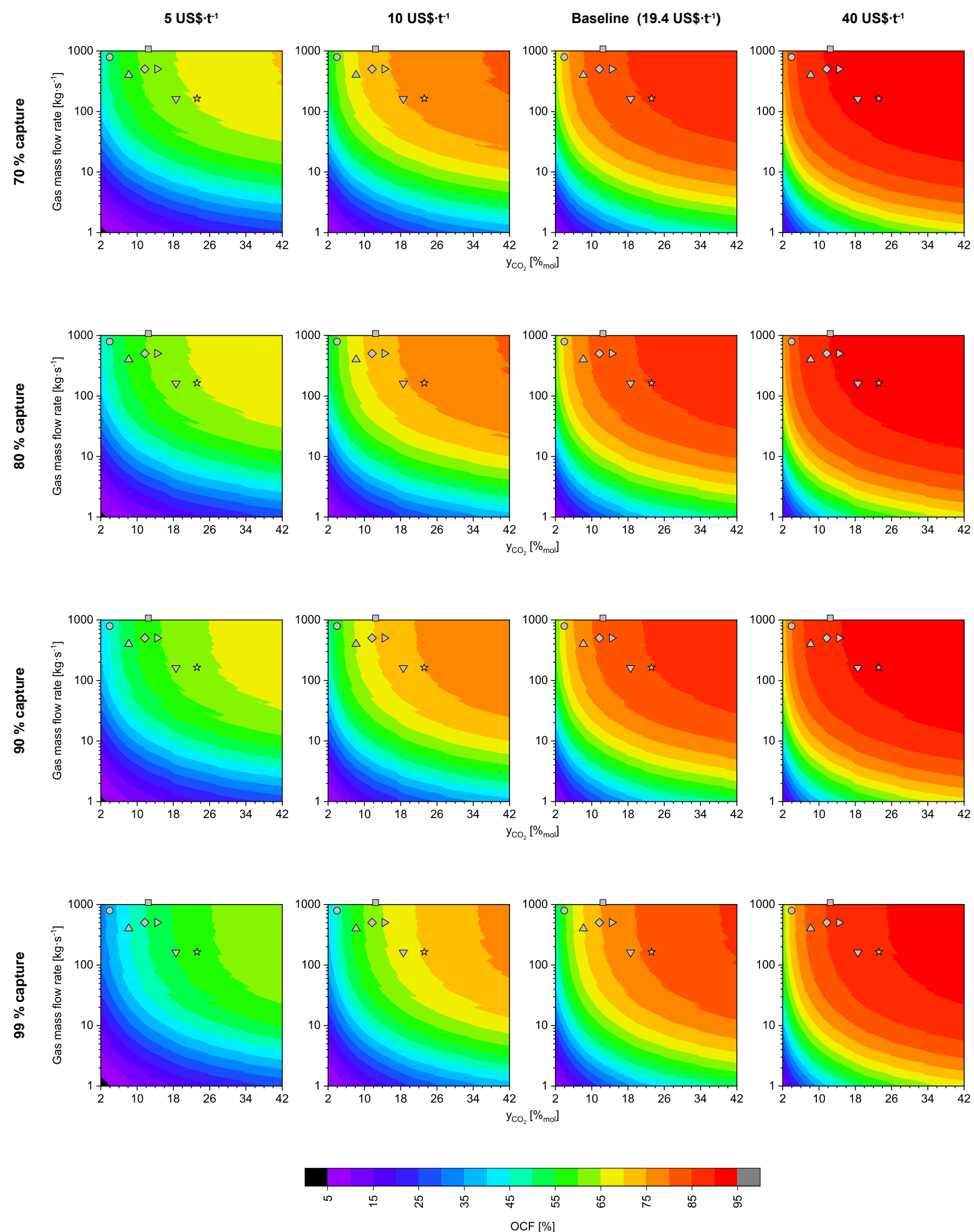

○ 500 MW NGCC $\triangle 500$ MW NGCC $50 \%$ EGR $\diamond 500$ MW high-rank PC $\square 500$ MW low-rank PC $\triangleright 500 \mathrm{MW}$ biomass $\nabla 1 \mathrm{MMtpa}$ cement मे $1 \mathrm{MMtpa}$ steel

Figure S6 - Effect of steam cost (columns) on operating cost fraction at four different capture rates (rows), $70 \%, 80 \%, 90 \%$, and $99 \%$. 


\section{S2.6. $\quad$ Effect of reduced SRD}

\section{S2.6.1. Cost of capture}
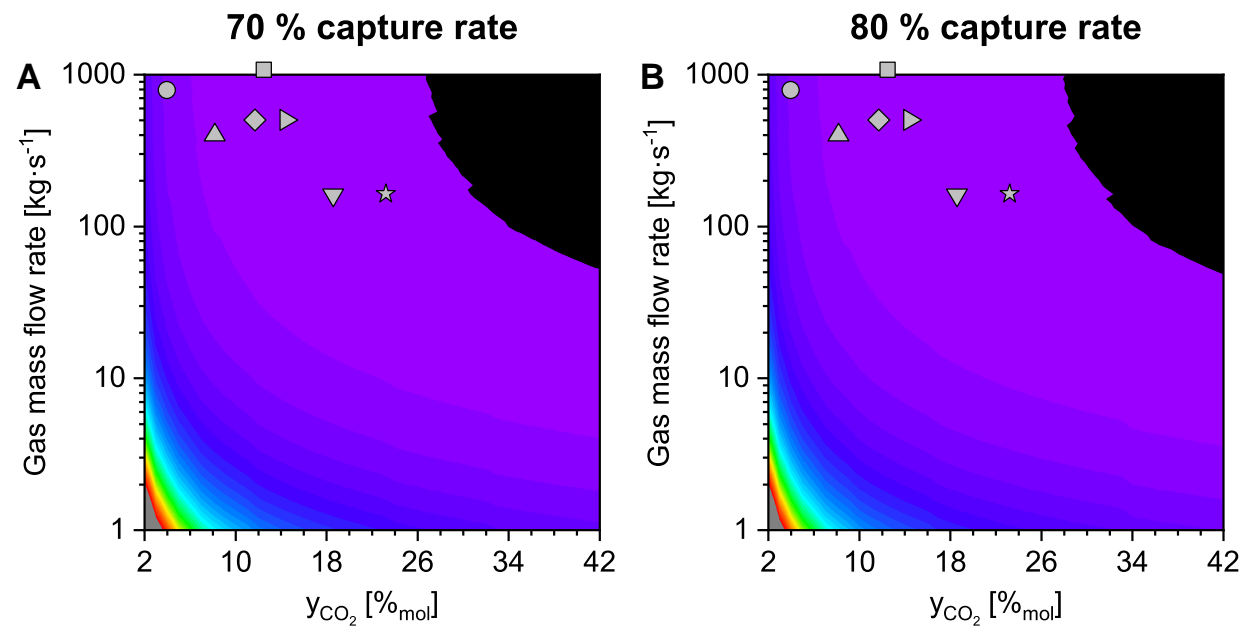

Capture cost
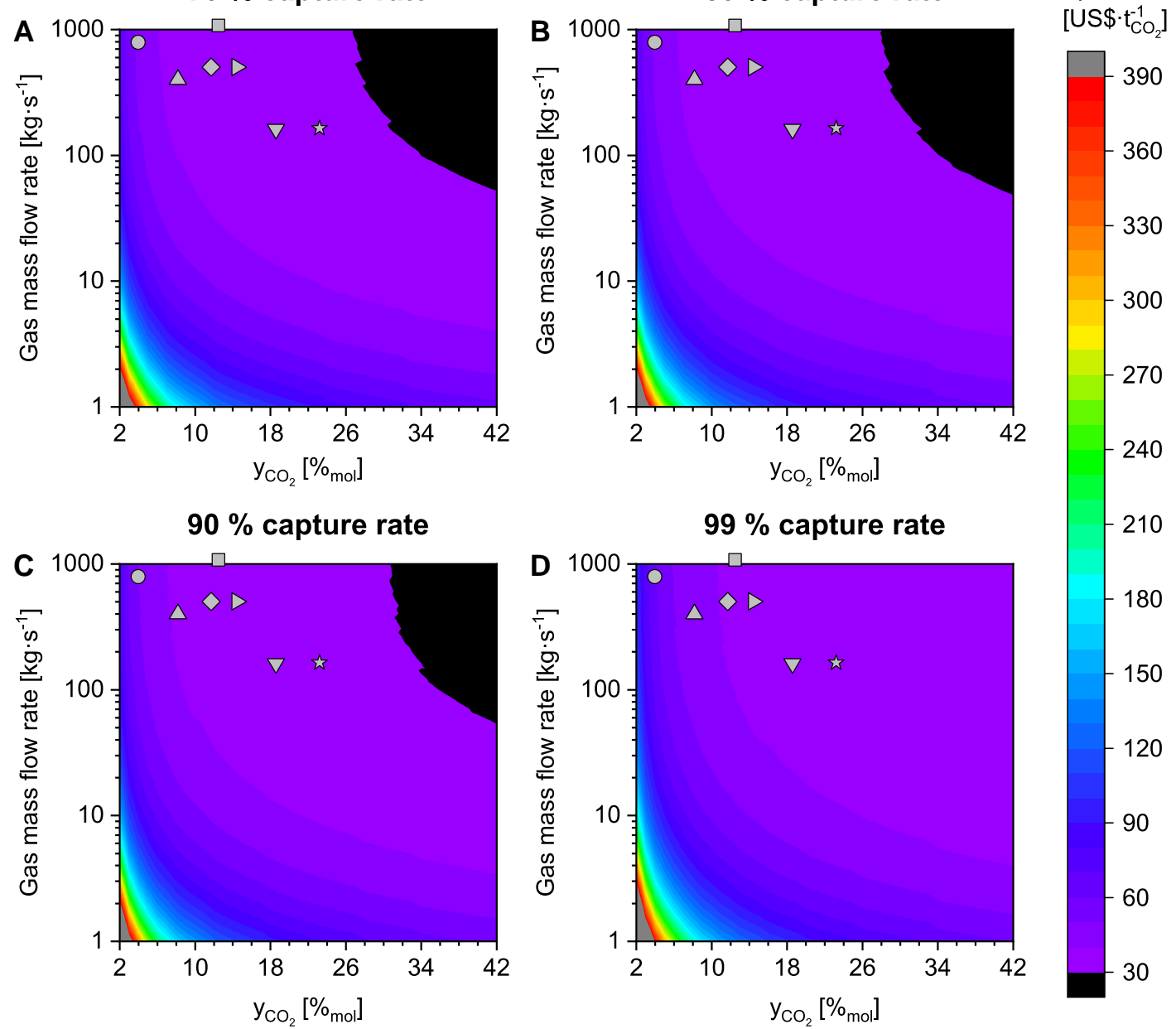

○ 500 MW NGCC $\triangle 500$ MW NGCC $50 \%$ EGR $\diamond 500$ MW high-rank PC $\square 500$ MW low-rank PC $\triangleright 500 \mathrm{MW}$ biomass $\nabla 1$ MMtpa cement is 1 MMtpa steel

Figure S7 - Effect of reduced regeneration energy requirements on cost of capture as a function of gas composition and flow rate at four different capture rates, 70\%, 80\%, 90\%, and $99 \%$. 
Table S1 - Capture costs in US\$·t $\mathrm{CO}_{2}{ }^{-1}$ at different capture rates for the reference post-combustion capture applications, at reduced regeneration energy requirements.

\begin{tabular}{|c|c|c|c|c|c|c|c|}
\hline \multirow{3}{*}{ Application } & \multirow{3}{*}{$\begin{array}{c}\text { ycO } 2 \\
{\left[\% \%_{\mathrm{mol}}\right]}\end{array}$} & \multirow{3}{*}{$\begin{array}{c}\text { Flow rate } \\
{\left[\mathrm{kg} \cdot \mathrm{s}^{-1}\right]}\end{array}$} & \multicolumn{5}{|c|}{ Capture cost $\left[\mathrm{US} \$ \cdot \mathrm{t}_{\mathrm{CO}^{-1}}{ }^{-1}\right]$} \\
\hline & & & \multicolumn{5}{|c|}{ Capture Rate [\%] } \\
\hline & & & 70 & 80 & 90 & 95 & 99 \\
\hline 500 MW NGCC & 3.98 & 791 & 47.1 & 47.7 & 50.1 & 53.1 & 61.8 \\
\hline 500 MW NGCC 50\% EGR & 8.19 & 401 & 37.5 & 37.8 & 38.9 & 40.4 & 44.5 \\
\hline 500 MW high-rank coal & 11.7 & 503 & 34.2 & 34.5 & 35.4 & 36.5 & 39.3 \\
\hline 500 MW low-rank coal & 12.5 & 1077 & 33.4 & 33.8 & 34.6 & 35.5 & 38.1 \\
\hline $500 \mathrm{MW}$ biomass & 14.4 & 503 & 32.8 & 33.1 & 33.7 & 34.5 & 36.8 \\
\hline 1 MMtpa cement & 18.6 & 162 & 32.2 & 32.3 & 32.7 & 33.4 & 35.1 \\
\hline 1 MMtpa steel & 23.2 & 164 & 31.2 & 31.2 & 31.6 & 32.1 & 33.7 \\
\hline
\end{tabular}

S2.6.2. Reduction in capture cost
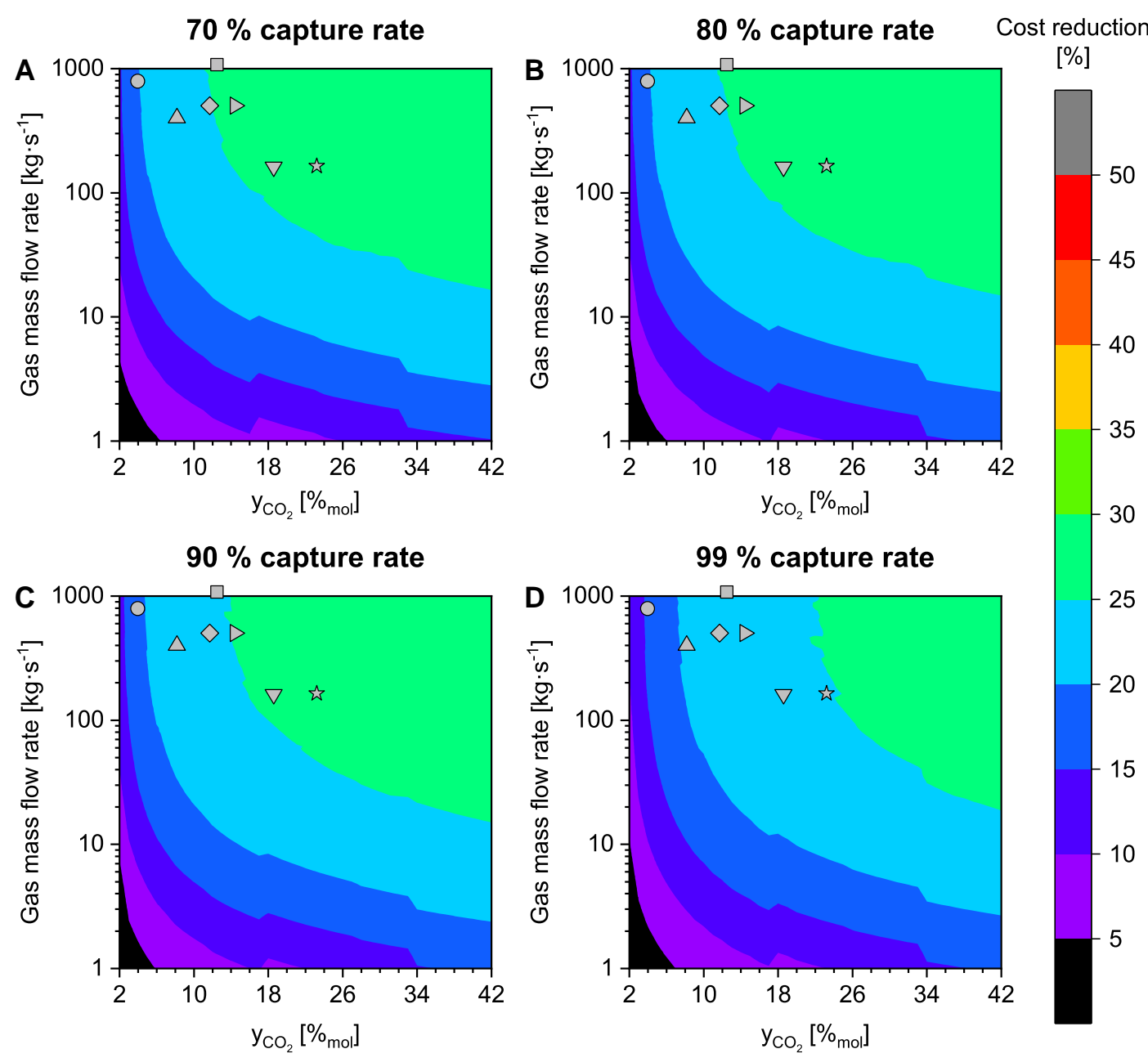
○ $500 \mathrm{MW}$ NGCC $\triangle 500 \mathrm{MW}$ NGCC $50 \%$ EGR $\diamond 500 \mathrm{MW}$ high-rank PC $\square 500 \mathrm{MW}$ low-rank PC $\triangleright 500 \mathrm{MW}$ biomass $\nabla 1 \mathrm{MMtpa}$ cement मे $1 \mathrm{MMtpa}$ steel

Figure S8 - Percentage cost reduction relative to MEA due to reduced regeneration energy requirements as a function of gas composition and flow rate at four different capture rates, 70\%, 80\%, 90\%, and 99\%. 
S2.6.3. Total annualised cost and operating cost fraction

Total annualised cost [MM US\$]

\section{Operating cost fraction [\%]}
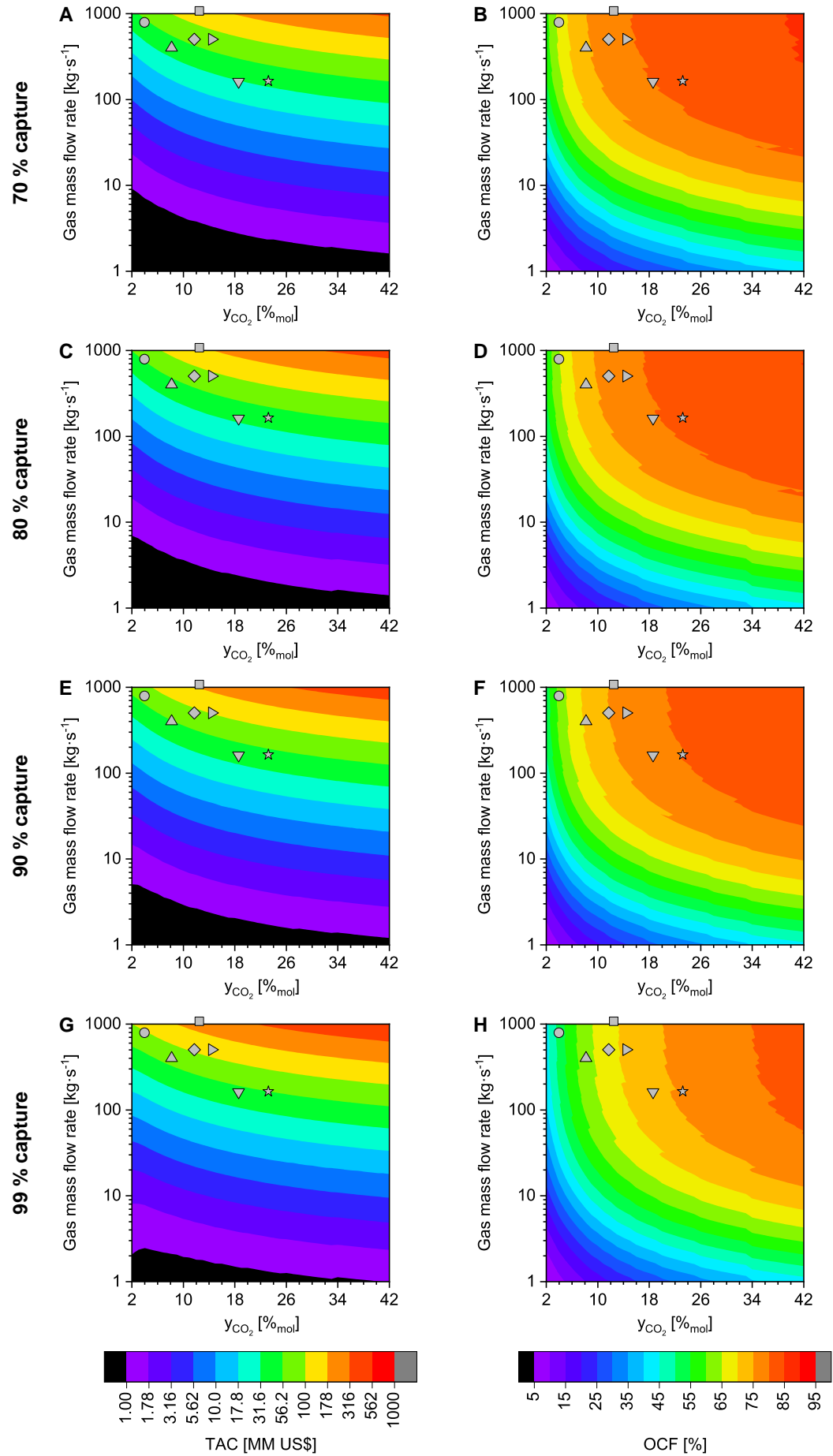

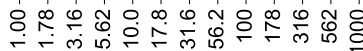
TAC [MM US\$]

OCF $[\%]$

○ 500 MW NGCC $\triangle 500$ MW NGCC $50 \%$ EGR $\diamond 500$ MW high-rank PC $\square 500$ MW low-rank PC $\triangle 500 \mathrm{MW}$ biomass $\nabla 1 \mathrm{MMtpa}$ cement $\& 1 \mathrm{MMtpa}$ steel

Figure S9 - Effect of reduced regeneration energy requirements on total annualized cost (A, C, E, G) and the operating cost fraction (B, D, F, H) at four different capture rates, $70 \%, 80 \%, 90 \%$, and $99 \%$. The colormap for the TAC plots (left column) uses a logarithmic scale. 
S2.7. Marginal cost as a function of flow rate - rescaled

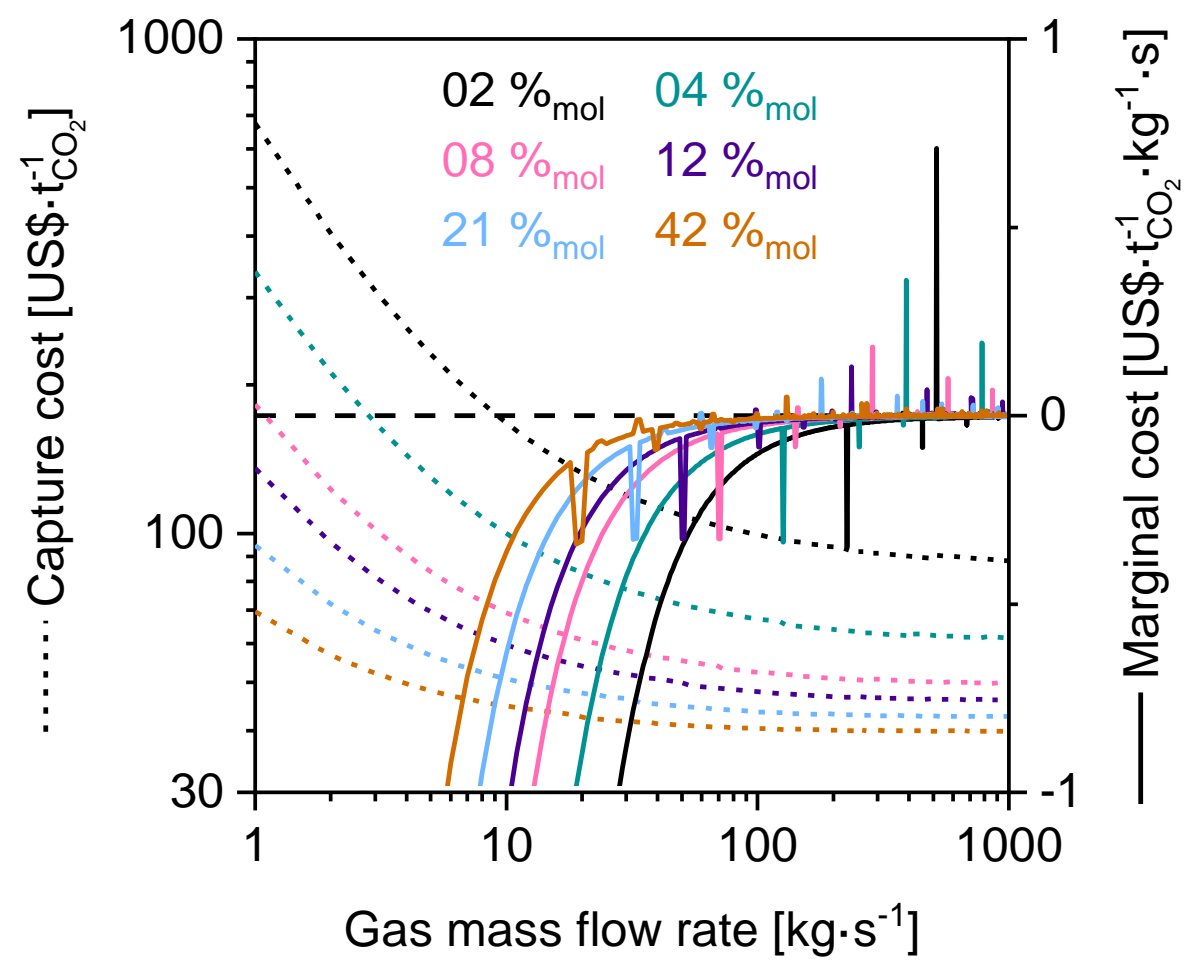

Figure S10 - Figure 3 rescaled to emphasise marginal cost (solid lines) changes as a function of flow rate for a fixed capture rate of $90 \%$. 


\section{S3. Surrogate model}

\section{S3.1. Methodology}

\section{S3.1.1. Model fitting}

A surrogate model (i.e. black-box model) consists of one or more algebraic expressions fit to data, which enables regression of that data. Data generated (described below) serves as the input, or training, data for the model. The data was fit using ALAMO. ${ }^{15}$ ALAMO generates 'data-driven' surrogate models by combining multiple function types to fit the data using its solver, BARON.

A data set was generated separate to that used in the main body of this work. It was desired to have the surrogate model a function of flow rate (1 to $1000 \mathrm{~kg}^{-1} \mathrm{~s}^{-1}, \mathrm{CO}_{2}$ concentration $\left(0.02\right.$ to $\left.0.42 \mathrm{~mol} \cdot \mathrm{mol}^{-1}\right)$, capture rate $\left(0.70\right.$ to $\left.0.99 \mathrm{~mol} \cdot \mathrm{mol}^{-1}\right)$, interest rate $\left(0.02\right.$ to $0.20 \$ \$ \$$ ), steam cost ( 1 to $\left.50 \mathrm{US} \$ \cdot \mathrm{t}^{-1}\right)$, and electricity cost (50 to $200 \mathrm{US} \$ \mathrm{MWh}^{-1}$ ). The amine lean loading was set to a constant value of 0.27 mol $_{\mathrm{CO} 2} \cdot \mathrm{mol}_{\mathrm{MEA}}{ }^{-1}$, as per the data in the main body of the work. Latin hypercube sampling was used to generate a set of 12000 points to cover the ranges stipulated.

\section{S3.1.2. Error analysis}

Aside from the traditional parity and residual plots, the goodness-of-fit metrics for the surrogate model are also provided. Statistical analysis is provided on the basis of absolute and percentage error.

The parameters are defined as below, where $\mathrm{y}_{\mathrm{a}}$ is the 'actual value' or 'input value', and $\mathrm{yf}_{\mathrm{f}}$ is the 'fitted value' or 'predicted value'.

$$
\begin{aligned}
& \text { Residual }=y_{f}-y_{a} \\
& \operatorname{Err}_{\text {absolute }}=\left|y_{f}-y_{a}\right| \\
& \text { Err }_{\text {percentage }}=\left|\frac{y_{f}-y_{a}}{y_{a}}\right| \times 100
\end{aligned}
$$

\begin{tabular}{|c|c|c|}
\hline Number of points & Number of input points to model & \\
\hline Minimum & Minimum value of $\operatorname{Err}_{\text {absolute }}$ & \multirow{5}{*}{ [units] } \\
\hline Maximum & Maximum value of Err $_{\text {absolute }}$ & \\
\hline Average & Average value of Err $_{\text {absolute }}$ & \\
\hline Median & Median value of Err ${ }_{\text {absolute }}$ & \\
\hline Standard deviation & Standard deviation of Errabsolute & \\
\hline
\end{tabular}

Absolute error statistics (example table) 
The percentage error statistic table shows the number of points (and the corresponding percentage of the total number of points) of the dataset that have percentage errors greater than the indicated value. The table is not bounded, i.e. if 17 points have a percentage error greater than $5 \%$, and 4 points greater than $10 \%$, there are 13 points between 5 and $10 \%$.

\section{Percentage error statistics (example table)}

\begin{tabular}{|l|c|c|}
\hline $\begin{array}{l}\text { Percentage } \\
\text { error }\end{array}$ & $\begin{array}{c}\text { Number of points } \\
\text { greater than }\end{array}$ & $\begin{array}{c}\text { Percentage of total } \\
\text { points }\end{array}$ \\
\hline 5 & & \\
\hline 10 & & \\
\hline 15 & & \\
\hline 20 & & \\
\hline
\end{tabular}




\section{S3.2. Goodness-of-fit}
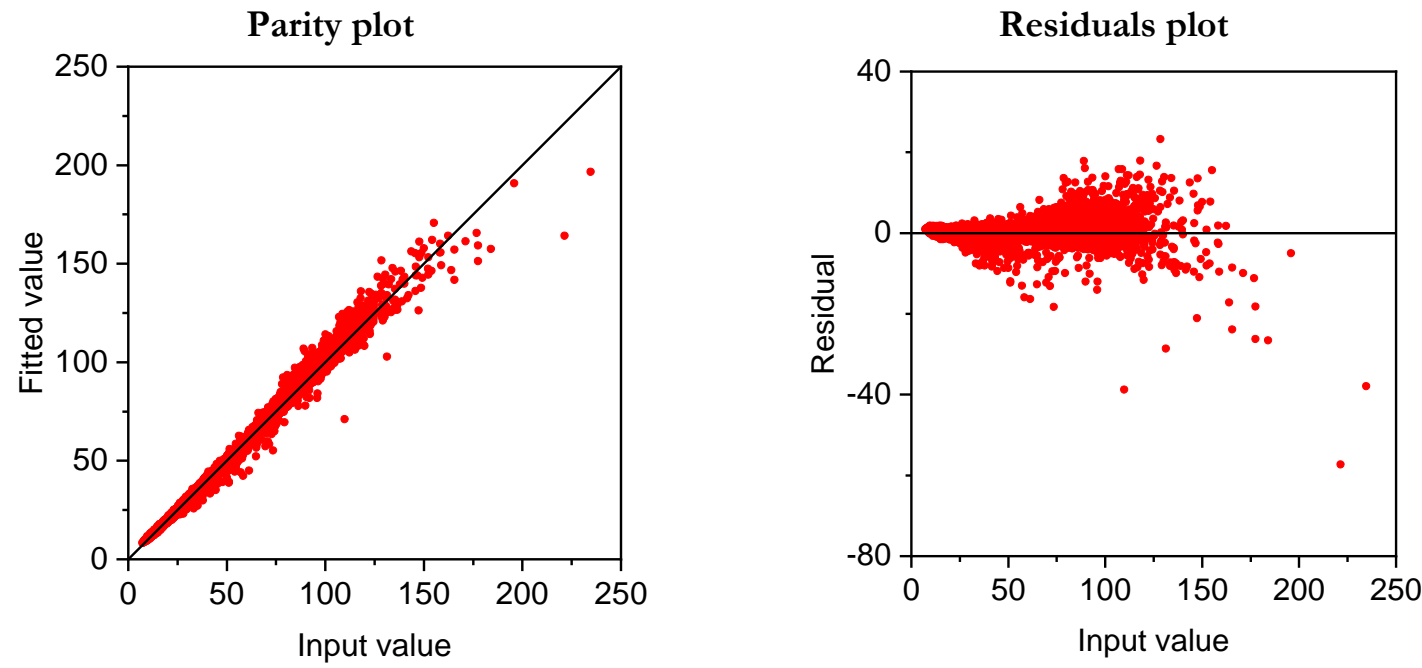

Figure S11 - Parity and residuals plots of the surrogate model fit.

$\underline{\text { Absolute error statistics }}$

Table S2 - Absolute error statistics of the surrogate model fit.

\begin{tabular}{|l|c|c|}
\hline Number of points & 12000 & \\
\cline { 1 - 2 } Minimum & $2.04 \cdot 10^{-5}$ & \\
\cline { 1 - 2 } Maximum & $5.73 \cdot 10^{1}$ & \multirow{2}{*}{$\mathrm{US} \$ \cdot \mathrm{tCO}^{-1}$} \\
\cline { 1 - 2 } Average & 1.25 & \\
\cline { 1 - 2 } Median & $7.68 \cdot 10^{-1}$ & \\
\cline { 1 - 2 } Standard deviation & 1.80 & \\
\hline
\end{tabular}

\section{$\underline{\text { Percentage error statistics }}$}

Table S3 - Percentage error statistics of the surrogate model fit.

\begin{tabular}{|l|c|c|}
\hline $\begin{array}{l}\text { Percentage } \\
\text { error }\end{array}$ & $\begin{array}{c}\text { Number of points } \\
\text { greater than }\end{array}$ & $\begin{array}{c}\text { Percentage of total } \\
\text { points }\end{array}$ \\
\hline $\mathbf{5}$ & 962 & 8.02 \\
\hline $\mathbf{1 0}$ & 181 & 1.51 \\
\hline $\mathbf{1 5}$ & 46 & $3.83 \cdot 10^{-1}$ \\
\hline $\mathbf{2 0}$ & 15 & $1.25 \cdot 10^{-1}$ \\
\hline
\end{tabular}




\section{S4. Equipment size limits}

The same size limitations apply from the original implementation of the model ; however, the key information is summarised here. Beyond these limits, equipment is parallelised.

Table S4 - Unit operation size limits

\begin{tabular}{|l|c|}
\hline Unit operation & Size limit \\
\hline Absorber & diameter $\leq 16 \mathrm{~m}$ \\
\hline Stripper & diameter $\leq 16 \mathrm{~m}$ \\
\hline Feed gas blower & $1000 \mathrm{~m}^{3} \cdot \mathrm{s}^{-1}$ \\
\hline Heat exchangers & $1500 \mathrm{~m}^{2}$ \\
\hline
\end{tabular}

\section{S5. Model updates}

\section{S5.1. Pressure drop}

The published model utilised the GPDC pressure drop correlation for structured packing.

For this work it has been replaced with the hydraulic model published by Rocha et al. for structured packing. ${ }^{16}$ This was in order to widen the range of applicability.

\section{S5.2. Absorber rich amine outlet temperature}

The published model performed an energy balance based on the 2 inlet and 2 outlet streams accounting for the heat of reaction and heat capacities of the streams. The rich amine outlet temperature was the degree of freedom.

The result of this was that the "temperature bulge" was inadvertently placed at the bottom of the absorber. The consequences of this were that the attainable rich loadings were much lower than published pilot plant and detailed simulation data, i.e. more concentrated feed gas streams were not being taken advantage of.

A compromise was opted for whereby the rich amine outlet temperature is now a simple function of the gas inlet temperature. This was based on TCM pilot plant data where the temperature profiles showed that the rich amine outlet temperature was between 2 and $5 \mathrm{~K}$ above the feed gas temperature. ${ }^{10}$ The rich amine outlet temperature is now given by:

$$
\mathrm{T}_{\text {rich,out }}=\mathrm{T}_{\text {gas,feed }}+2
$$

The limitation of this approach are that at high $\mathrm{L} / \mathrm{G}$ ratios, i.e. those experienced above a $\mathrm{ycO}_{\mathrm{CO}}$ of $12-15$ $\%$ mol, the thermal mass of the down coming liquid is sufficiently large such that the feed gas flow will not 
be able to cool the liquid as effectively. Therefore, this new approach may progressively overestimate the rich loading at feed $\mathrm{CO}_{2}$ concentrations above the mentioned range.

\section{S5.3. Stripper outlet gas temperature}

In the published version of the model a reflux ratio was assumed in order to calculate obtain the overhead temperature of the stripper.

For this model, it is assumed that the gas exiting the stripper is in thermal equilibrium with the inlet liquid.

\section{S5.4. Capital cost estimation}

As mention in the methods section, the IChemE factorial cost estimation method has been replaced with the Lang factor method. A Lang factor of 3.6 has been applied.

The consequences of this are addressed in the paper's methods section.

\section{S5.5. Vapour liquid equilibrium (VLE)}

The originally published model utilised the equilibrium model for MEA proposed by Gabrielsen et al. ${ }^{17}$ The limitation of this model was two-fold for this work. It was not possible to achieve amine loadings above $\approx 0.46$, and this was not able to represent the rich loadings that would be obtained with high $\mathrm{CO}_{2}$ partial pressure flue gases. The other is that it is not a speciated model, and as will be discussed later, the concentration of some species in solution were required for the updated mass transfer calculation methods.

The $\mathrm{CO}_{2}-$ MEA VLE is now described by the model prepared by Park et al. ${ }^{18}$ The limitation of this model is that it cannot be applied below $303 \mathrm{~K}$.

Another requirement of the updated mass transfer calculation methods was for gas and liquid phase compositions. Therefore, the Park model has also been incorporated into a multicomponent VLE model, the Park model assumes an ideal liquid phase and this is carried over to the multicomponent VLE model. The system of equations is solved in MATLAB using the lsqnonlin algorithm in order to apply lower and upper bounds to the solutions, to a tolerance of $10^{-18}$. The equations are formulated in terms of the logarithms of the variables in order to facilitate reliable solutions, as the variables span many orders of magnitude (from $10^{-14}$ to $10^{1}$ ).

The model is used in two ways: 
1. The system T, P, and gas phase $\mathrm{yCO}_{\mathrm{CO}}$ can be specified - the other gas phase components and the liquid phase species are solved.

2. The system $\mathrm{T}, \mathrm{P}$, amine loading, and $\mathrm{N}_{2}$ dissolved in the liquid phase can be specified - the complete gas phase, and liquid phase species are solved.

Case 1 will be described, and the variations for case 2 will be mentioned.

\section{S5.5.1. Case $1-\mathrm{T}, \mathrm{P}, \mathrm{ycO}_{\mathrm{CO}}$ are specified}

The first 7 equations are used directly from Park et al. without modification. ${ }^{18}$ Park et al. also provide a method in their paper to estimate reliable initial guesses to begin the solution. Square brackets represent the liquid phase concentration in $\mathrm{mol} \cdot \mathrm{L}^{-1}$.

\begin{tabular}{|c|c|c|}
\hline 1. & $K_{A}=\frac{\left[R N H_{2}\right]\left[H^{+}\right]}{\left[R H^{+}\right]}$ & \\
\hline & {$\left[R N H_{3}^{+}\right]$} & \\
\hline \multirow{2}{*}{2.} & \multirow{2}{*}{\multicolumn{2}{|c|}{$K_{C}=\frac{\left[\mathrm{RNHCOO}^{-}\right]}{\left[\mathrm{RNH}_{2}\right]\left[\mathrm{HCO}_{3}^{-}\right.}$}} \\
\hline & & \\
\hline 3. & \multicolumn{2}{|l|}{$K_{3}=\frac{\left[\mathrm{H}^{+}\right]\left[\mathrm{HCO}_{3}^{-}\right]}{\left[\mathrm{CO}_{2}\right]}$} \\
\hline 4. & \multicolumn{2}{|l|}{$K_{4}=\frac{\left[\mathrm{H}^{+}\right]\left[\mathrm{CO}_{3}^{2-}\right]}{\left[\mathrm{HCO}_{3}^{-}\right]}$} \\
\hline 5. & \multicolumn{2}{|l|}{$K_{5}=\left[\mathrm{H}^{+}\right]\left[\mathrm{OH}^{-}\right]$} \\
\hline 6. & \multicolumn{2}{|c|}{$[\mathrm{MEA}]=\left[\mathrm{RNH}_{2}\right]+\left[\mathrm{RNH}_{3}^{+}\right]+\left[\mathrm{RNHCOO}^{-}\right]$} \\
\hline 7. & {$\left[H^{+}\right]+\left[R N H_{3}^{+}\right]=[R$} & $\left.\mathrm{NHCOO}^{-}\right]+\left[\mathrm{HCO}_{3}^{-}\right]+2\left[\mathrm{CO}_{3}^{2-}\right]+\left[\mathrm{OH}^{-}\right]$ \\
\hline 8. & \multicolumn{2}{|c|}{$\phi_{\mathrm{CO}_{2}} y_{\mathrm{CO}_{2}} \mathrm{P}=\gamma_{\mathrm{CO}_{2}}\left[\mathrm{CO}_{2}\right] \mathrm{H}_{\mathrm{CO}_{2}}$} \\
\hline 9. & \multicolumn{2}{|c|}{$\phi_{N_{2}} y_{N_{2}} P=\gamma_{N_{2}}\left[N_{2}\right] H_{N_{2}}$} \\
\hline 10. & $\phi_{W} y_{W} P=a_{W} P_{W}^{\circ} \phi_{W}^{\circ} \cdot \exp$ & {$\left[\frac{V_{W}^{\circ} \cdot\left(P-P^{\circ}\right)}{R \cdot T}\right.$} \\
\hline 11. & $y_{\mathrm{CO}_{2}}+y_{N_{2}}+y_{W}=1$ & \\
\hline
\end{tabular}

Where $\varphi$ represents the fugacity coefficient of the component in the mixture, the Peng-Robinson EOS is used to calculate the gas phase fugacity coefficients. ${ }^{19}$ The binary interaction parameters were calculated based on the method proposed by Qian et al. ${ }^{20}$ Physical properties and constants were taken from Poling. ${ }^{21}$ 
For equation $10, a_{w}$ represents the activity of water, which in this case is set to its mole fraction. $\mathrm{P}^{\circ} \mathrm{w}, \varphi^{\circ} \mathrm{w}$, and $\mathrm{V}^{\circ}$ w represent the saturation pressure at the system temperature, and the vapour fugacity coefficient and liquid molar volume at the saturation conditions, for pure water, respectively.

The functions for the 5 equilibrium constants and the Henry constant for $\mathrm{CO}_{2}$ were provided in Park et al. $\mathrm{K}_{\mathrm{A}}$ and $\mathrm{K}_{\mathrm{C}}$ are required to be fit from the provided data points and the fits are provided below. Data is only provided down to $313 \mathrm{~K}$ and the fit for $\mathrm{K}_{\mathrm{C}}$ is not reliable below $303 \mathrm{~K}$, thus the imposed temperature limitation.

$\ln \left(K_{A}\right)=\frac{5310428}{T^{2}}-\frac{38043.5}{T}+45.1329$
$\ln \left(K_{C}\right)=\frac{-6636555}{T^{2}}+\frac{43749.8}{T}-68.745$

The Henry constant for $\mathrm{N}_{2}$ was taken from Table 5.4 in Burkholder et al. ${ }^{22}$

The $\mathrm{CO}_{2}$ loading of the solution is calculated once the system of equations is solved using the following equation:

$$
\alpha=\frac{\left[\mathrm{CO}_{2}\right]+\left[\mathrm{HCO}_{3}^{-}\right]+\left[\mathrm{RNHCOO}^{-}\right]+\left[\mathrm{CO}_{3}^{2-}\right]}{\left[\mathrm{RNH}_{2}\right]+\left[\mathrm{RNH}_{3}^{+}\right]+\left[\mathrm{RNHCOO}^{-}\right]}
$$

\section{S5.5.2. Case $2-\mathrm{T}, \mathrm{P}, \alpha,\left[\mathrm{N}_{2}\right]$ are specified}

In addition to the equations for case 1 , the following is added:

$$
[\mathrm{MEA}] \cdot \alpha=\left[\mathrm{CO}_{2}\right]+\left[\mathrm{HCO}_{3}^{-}\right]+\left[\mathrm{RNHCOO}^{-}\right]+\left[\mathrm{CO}_{3}^{2-}\right]
$$




\section{S5.5.3. Comparison}

The $\mathrm{CO}_{2}$ - MEA VLE is compared to Gabrielsen et al. ${ }^{17}$ and experimental data from Jou et al.23

$313.15 \mathrm{~K}$

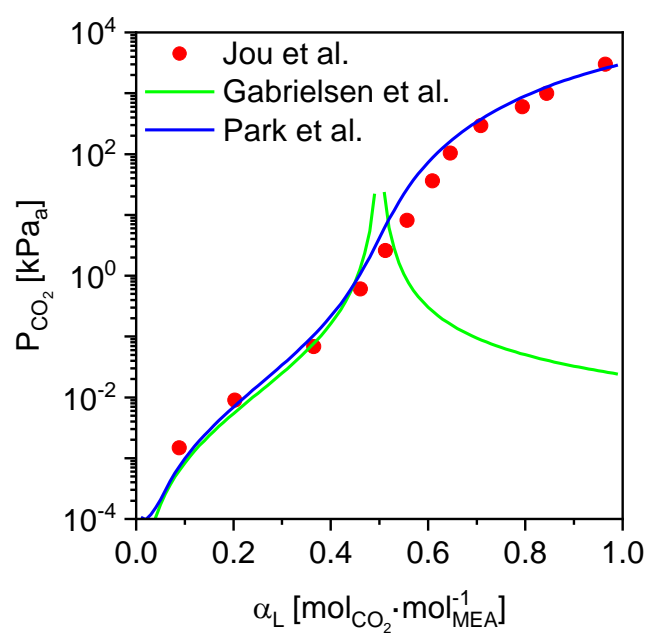

$353.15 \mathrm{~K}$

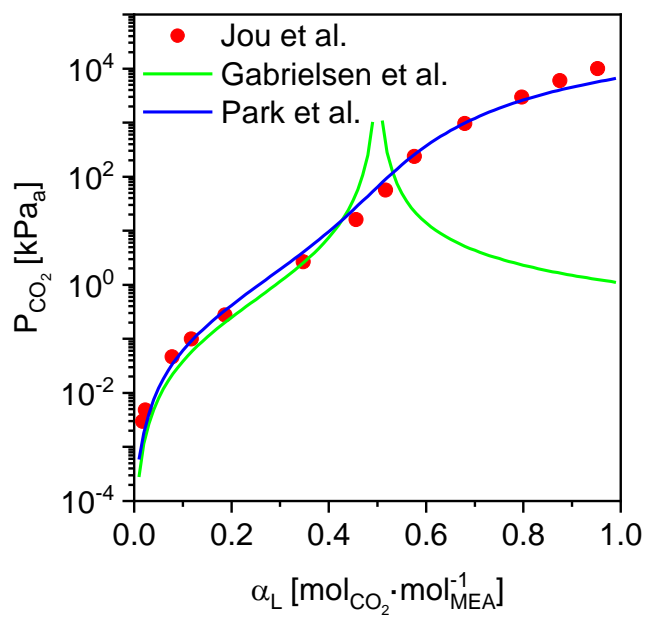

$333.15 \mathrm{~K}$

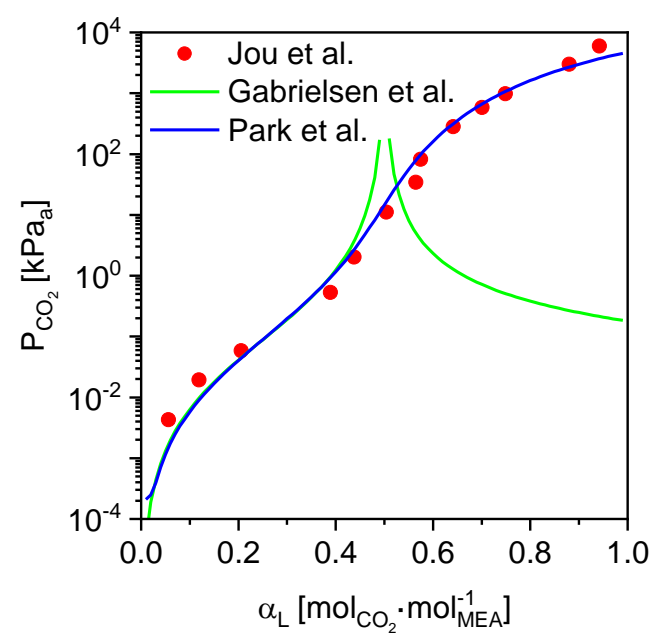

$373.15 \mathrm{~K}$

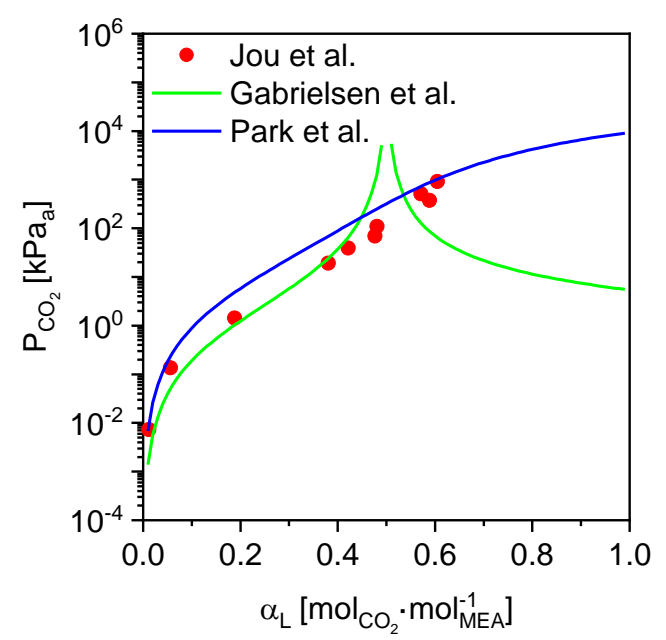

Figure S12 - Comparison between VLE models for the $\mathrm{CO}_{2}$ - MEA system.

\section{S5.6. $\quad$ Feed blower/compressor}

In the original model, the feed blower was either costed as a blower or compressor depending on the feed gas flow rate and duty. For this work, this crossover was removed as it was causing discontinuities in the cost profiles. The feed blower is now only costed using the compressor cost curve.

The difference in cost curves up to a flow rate of $15 \mathrm{~m}^{3} \cdot \mathrm{s}^{-1}$ are shown in Figure S13. In the original work, the changeover occurred at $15 \mathrm{~m}^{3} \cdot \mathrm{s}^{-1}$, as this is the maximum range of the cost data provided for the blower selected (turbo-blowers with maximum outlet pressure of $69 \mathrm{kPa} / 10 \mathrm{psi}$ ). ${ }^{24}$ As the compressor is costed based on input power, the specific work was calculated for a feed gas temperature of $313.15 \mathrm{~K}$, 
pressure ratio of $1.09, \mathrm{C}_{\mathrm{P}} / \mathrm{C}_{\mathrm{V}}$ of 1.38 , and isentropic efficiency of 0.83 , for a gas with molecular weight $29.26 \mathrm{~g} / \mathrm{mol}$ and $1.12 \mathrm{~kg} / \mathrm{m}^{3}$ density.

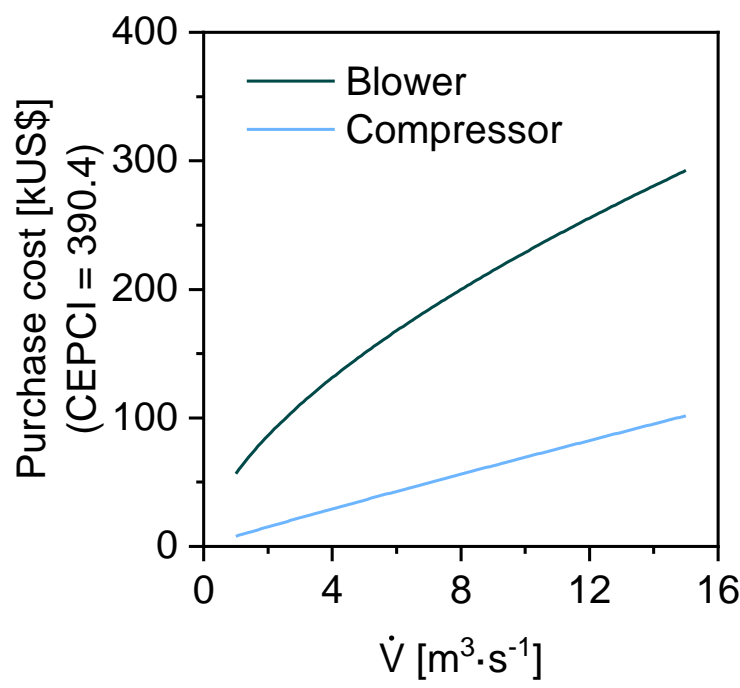

Figure S13 - Installed cost curves for a blower and compressor as a function of inlet volumetric flow rate.

The minimum size specified in the costing reference for a motor-driven centrifugal compressor is $75 \mathrm{~kW}$, ${ }^{24}$ which in the scenario above corresponds to a flow rate of $7.3 \mathrm{~m}^{3} \cdot \mathrm{s}^{-1}$. Values below this are therefore extrapolations. Although compressors are unlikely to be used as feed blowers in this application, a compromise had to be made based on the costing data available. The large axial fans used in power plant applications are able to move $\sim 1000 \mathrm{~m}^{3} \cdot \mathrm{s}^{-1}$ of flue gas at pressure increases of up to $20-30 \mathrm{kPa}$. Given the power consumption of such an application, and as the construction/assembly of these units is analogous to axial centrifugal compressors, it was decided to cost the feed blower as a compressor for the higher flow rate applications rather than extrapolate the blower cost curve.

Therefore, relative to the full implementation of the model without this simplification, the capital cost of the lower flow rate applications are underestimated.

\section{S5.7. Stripper bottom pressure}

In order to automate the data collection as best as possible, it was necessary to select a stripper operating pressure that did not result in flashing of the inlet liquid under the wide range of operating conditions. In order to enable this, the pressure is set as a function of amine lean loading according to the relationship below. This yields a pressure of 3 bar $_{a}$ at a lean loading of 0.10 , and 1.8 bara $_{a}$ at a lean loading of 0.35 .

$P_{\mathrm{Str}}^{\mathrm{btm}}=-4.8 \times \alpha_{L L}+3.48$ 


\section{S5.8. Stripper overhead pressure drop}

The pressure drop between the stripper outlet and condenser was originally set to 2 psi $(13.8 \mathrm{kPa})$.

It is now set to $5 \mathrm{kPa}$ to also match the TCM pilot plant data.

\section{S5.9. Reboiler temperature margin}

The reboiler and stripper bottom temperatures were originally set based on the temperature required to achieve the desired lean loading.

An additional margin $(10 \mathrm{~K})$ is now added to account for heat losses, in line with the TCM pilot plant results.

\section{S5.10. Thermophysical properties of water}

The range of applicability has been widened for the saturation temperature and pressure of water. The data is taken from the same source, the NIST Thermophysical Properties of Fluid Systems database.

The new functions are as follows:

$T^{\text {sat }}=\left[-1.06987541 \cdot 10^{-6} \times \ln (P)^{2}-1.06170745 \cdot 10^{-4} \times \ln (P)+1.78188927 \cdot 10^{-3}\right]^{-1}-459.67$

Where $0.1<\mathrm{P}<70.1 \mathrm{psia}$, and $\mathrm{T}^{\text {sat }}$ is in ${ }^{\circ} \mathrm{F}$.

The agreement between this function and datapoints from the NIST database is shown in Figure S14.

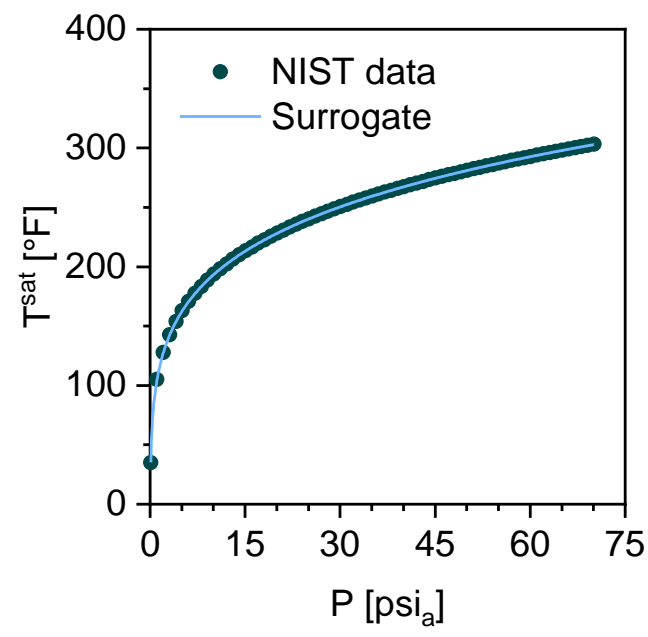

Figure S14 - Comparison between $\mathrm{T}^{\text {sat }}$ data of water and output from surrogate function over the applicable pressure range. 


$$
\begin{aligned}
& x=\frac{1}{T+459.67} \\
& P^{\text {sat }}=\exp \left(-811558.51 x^{2}-6525.5445 x+14.201555\right)
\end{aligned}
$$

Where $35<\mathrm{T}<350^{\circ} \mathrm{F}$, and Psat is in psia.

The agreement between this function and datapoints from the NIST database is shown in Figure S15.

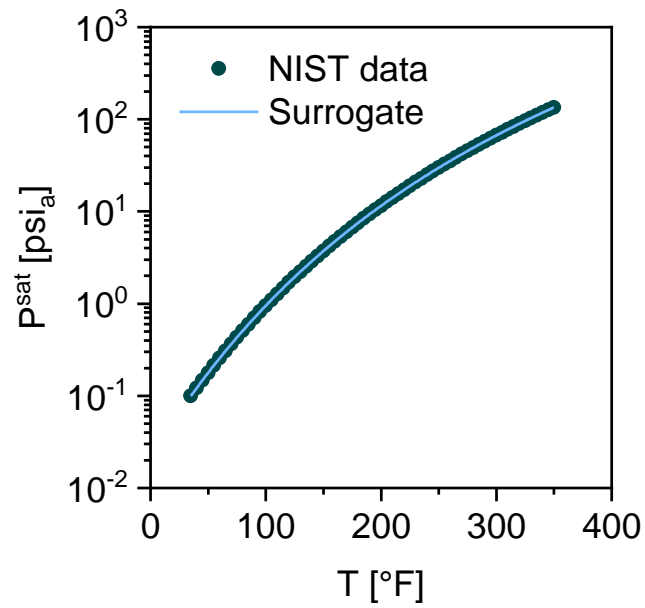

Figure S15 - Comparison between Psat data of water and output from surrogate function over the applicable temperature range.

\section{S5.11. Flue gas humidity}

In the original model, the flue gas was assumed to be dry in order to compare costs to an adsorption process.

Water content of the flue gas is now considered, and the water balances are accordingly.

The flue gas composition $\mathrm{y}_{\mathrm{CO} 2}$, is the wet $\mathrm{CO}_{2}$ composition.

The $\mathrm{CO}_{2}$ concentration is specified, the gas is saturated with water at the feed gas temperature, and the balance is nitrogen. This is undertaken based on a multicomponent VLE calculation, the following four equations are solved using the PR-EOS and corresponding method described in the $\mathrm{CO}_{2}$-MEA VLE section above. 


\begin{tabular}{|l|l|}
\hline 1. & $\phi_{\mathrm{CO}_{2}} y_{\mathrm{CO}_{2}} P=\gamma_{\mathrm{CO}_{2}}\left[\mathrm{CO}_{2}\right] \mathrm{H}_{\mathrm{CO}_{2}}$ \\
\hline 2. & $\phi_{\mathrm{N}_{2}} y_{\mathrm{N}_{2}} P=\gamma_{N_{2}}\left[N_{2}\right] H_{N_{2}}$ \\
\hline 3. & $\phi_{W} y_{W} P=a_{W} P_{W}^{\circ} \phi_{W}^{\circ} \cdot \exp \left[\frac{V_{W}^{\circ} \cdot\left(P-P^{\circ}\right)}{R \cdot T}\right]$ \\
\hline 4. & $y_{\mathrm{CO}_{2}}+y_{N_{2}}+y_{W}=1$ \\
\hline
\end{tabular}

\section{S5.12. Thermophysical properties of feed gas}

As the feed gas is now humid, the $\mathrm{k}$ value $\left(\mathrm{C}_{\mathrm{P}} / \mathrm{C}_{\mathrm{V}}\right)$ function was updated to include water. This function is used to calculate the isentropic work required for the feed gas blower.

The new function is below and returns $k$ for saturated mixture of $\mathrm{CO}_{2}-\mathrm{H}_{2} \mathrm{O}, \mathrm{N}_{2}-\mathrm{H}_{2} \mathrm{O}$, or $\mathrm{CO}_{2}-\mathrm{N}_{2}-\mathrm{H}_{2} \mathrm{O}$. The data was generated in HYSYS using the PR-EOS and fit using ALAMO.

$$
\begin{aligned}
k= & -2.0386 \times 10^{-3} T+2.4802 \times 10^{-5} P-0.10934 y_{C_{2}}+0.042192 y_{N_{2}}+0.13518 \cdot \ln (T) \ldots \\
& +2.1006 \times 10^{-3} \cdot \ln (P)+2.1244 \times 10^{-6} T^{2}+0.052207 y_{C_{2}}^{2}-0.0026856 y_{N_{2}}^{2} \ldots \\
& -9.8304 \times 10^{-3} y_{\mathrm{CO}_{2}}^{3}+6.4244 \times 10^{-3} y_{N_{2}}^{3}-1.7153 \times 10^{-8}\left(T \cdot y_{C_{2}}\right)^{2}+2.7449 \times 10^{-7}\left(T \cdot y_{N_{2}}\right)^{2} \ldots \\
& -5.0166 \times 10^{-3}\left(y_{\mathrm{CO}_{2}} \cdot y_{N_{2}}\right)^{2}+0.99054
\end{aligned}
$$

Where $90<\mathrm{P}<250 \mathrm{kPa}_{\mathrm{a}}, 293<\mathrm{T}<353 \mathrm{~K}, 0<\mathrm{yCO}_{2}<1,0<\mathrm{yN}_{\mathrm{N}}<1$

The goodness-of-fit plots showing the agreement between the input data points and the surrogate function are shown in Figure S16.
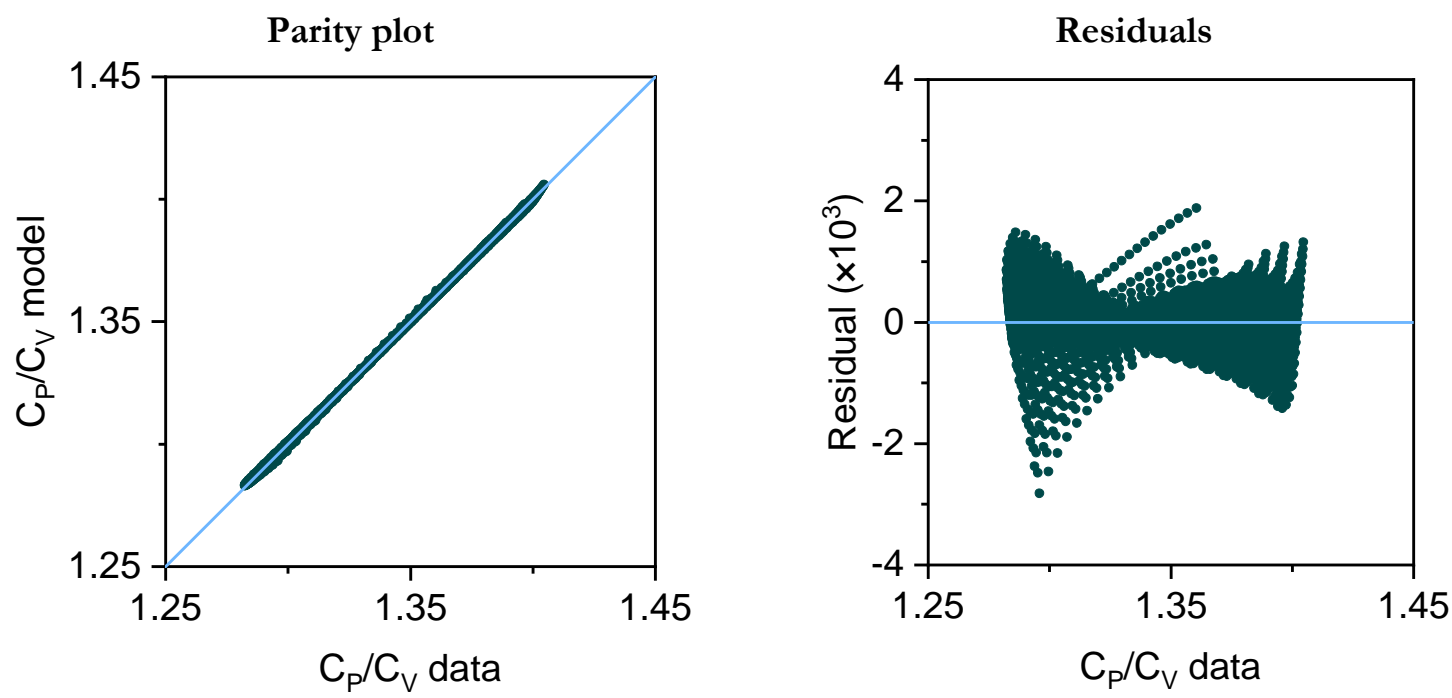

Figure S16 - Comparison between surrogate model and input data for the adiabatic index of the feed gas. Parity plot (left) and residuals plot (right). 
The feed gas density was originally calculated using an empirical correlation such as the above.

The feed gas density is now calculated using the PR-EOS for mixtures. As the compressibility is required to determine the fugacity coefficients for the feed humidity, this information is used to calculate the molar volume and hence density of the feed gas.

\section{S5.13. Wet acid gas heat capacity}

A wider range of applicability was required for this work. The function returns the heat capacity of a $\mathrm{CO}_{2}$ stream saturated with water at a given temperature and pressure. Data was generated in HYSYS using the PR-EOS and fit in ALAMO.

$$
\begin{aligned}
x= & T+459.67 \\
y= & 33.33817 \cdot \ln (x)-0.04351161 P^{2}-6.607161 \times 10^{-7} x^{3}+2.949299 \times 10^{-9} P^{5} \ldots \\
& +8.168338 \times 10^{-3}(x \cdot P)-2572.722 \frac{P}{x}+188544.2\left(\frac{P}{x}\right)^{3}-760262.1\left(\frac{P}{x}\right)^{4}-\frac{363.1274}{P} \\
C_{P}= & y^{\frac{-1}{\exp (1)}}
\end{aligned}
$$

Where $14<\mathrm{P}<70$ psia, $80<\mathrm{T}<295^{\circ} \mathrm{F}$, and $\mathrm{CP}$ is given in $\mathrm{BTU} \cdot 1 \mathrm{~b}^{-1 .{ }^{\circ}} \mathrm{F}^{-1}$.

The goodness-of-fit plots showing the agreement between the input data points and the surrogate function are shown in Figures S17 and S18.

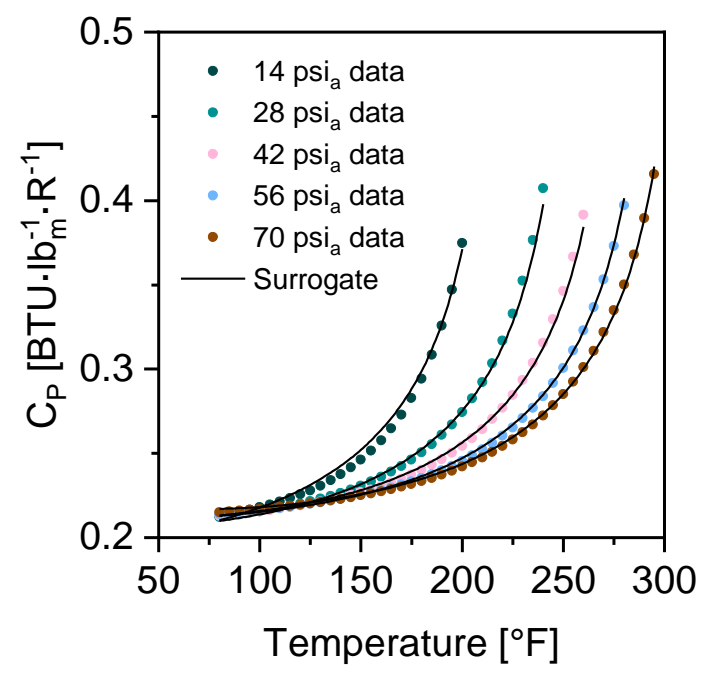

Figure S17 - Wet acid gas heat capacity data points at selected pressures over temperature compared with the surrogate model predictions. 

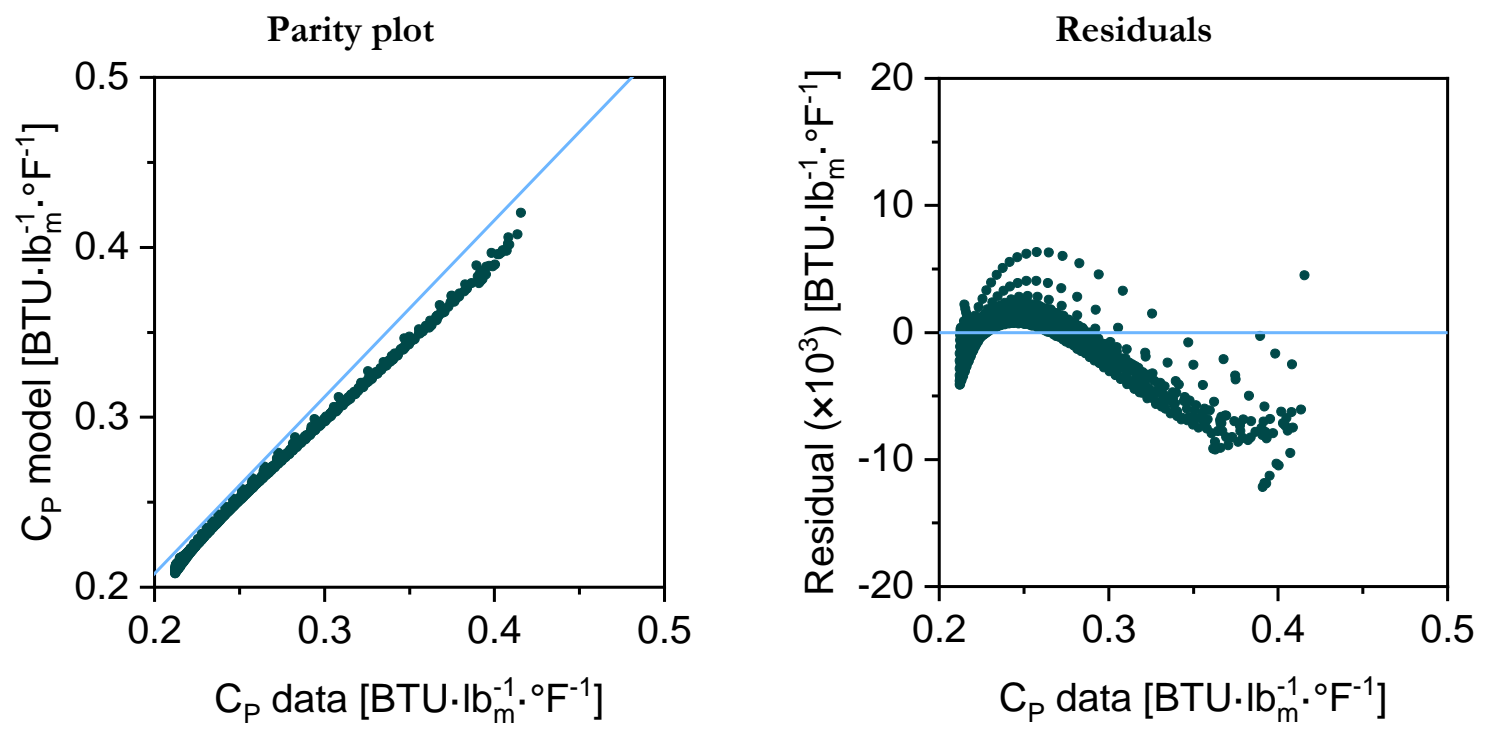

Figure S18 - Comparison between surrogate model and input data for the wet acid gas heat capacity. Parity plot (left) and residuals plot (right).

\section{S5.14. Rich amine physical properties}

The density and viscosity of the rich amine solution were required over a larger temperature range for this work. The original function was unsuitable over this range and thus an alternative approach has been used.

The properties are now determined using the methods proposed by Amundsen et al.25

\section{S5.15. Mass transfer}

In the original publication, the gas phase mass transfer resistance was ignored, and the same enhancement factor was used for the absorber and stripper. This was found to cause anomalies in the stripper dimensions under certain operating conditions, so the mass transfer approach has been updated for this work.

\section{S5.15.1. $\quad \mathrm{CO}_{2}$ liquid phase diffusivity}

The Wilke-Chang correlation was replaced with the method outlined in Ko et al. ${ }^{26}$ 


\section{S5.15.2. $\quad \mathrm{CO}_{2}$ gas phase diffusivity}

The two sets of binary diffusivity $\left(\mathrm{CO}_{2}-\mathrm{H}_{2} \mathrm{O}\right.$ and $\left.\mathrm{CO}_{2}-\mathrm{N}_{2}\right)$ were calculated based on the method outlined in Gilliland. ${ }^{27}$

The diffusivity of $\mathrm{CO}_{2}$ in the multicomponent mixture was estimated by the method outlined in Fairbanks and Wilke. 28

\section{S5.15.3. Mass transfer coefficients}

The gas and liquid phase mass transfer coefficients for structured packing were calculated using the method of Rocha et al.16, 29

\section{S5.15.4. Enhancement factors}

The enhancement factors for the absorber and stripper were taken from Harun et al..$^{30}$ There is no change for the absorber between the original version of the model and the one used in this work.

Additional data is required for the use of these enhancement factors. The liquid phase diffusivity of the carbamate ion was taken from Hoff $e t$ al. ${ }^{31}$ The liquid phase diffusivity of MEA was taken from Snijder $e t$ al. ${ }^{32}$ The $\mathrm{K}_{2}$ reaction equilibrium constant was fit from data in Jou $e t$ al. ${ }^{23}$ and is provided below.

$$
\ln \left(K_{2}\right)=\frac{-1308751}{T^{2}}+\frac{16164.47}{T}-28.8551
$$

\section{S5.15.5. General approach to flux calculation}

The $\mathrm{CO}_{2}$ flux is determined on the basis of fugacity/pressure:

$$
J=K_{G} \cdot\left(f_{\mathrm{CO}_{2}}^{\text {bulk }}-f_{\mathrm{CO}_{2}}^{\mathrm{int}}\right)
$$

The overall mass transfer coefficient $\left(\mathrm{K}_{\mathrm{G}}\right)$ is determined in the following way:

$$
K_{G}=\left[\frac{1}{k_{G}}+\frac{H_{\mathrm{CO}_{2}}}{k_{L} \cdot E}\right]^{-1}
$$

Where $\mathrm{E}$ is the enhancement factor.

For the absorber, the fugacity of $\mathrm{CO}_{2}$ at the interface is calculated using the following relation. ${ }^{33}$

$$
f_{\mathrm{CO}_{2}}^{\text {int }}=f_{\mathrm{CO}_{2}}^{\text {bulk }} \cdot\left[1+\frac{E \cdot k_{L}}{H \cdot k_{G}}\right]^{-1}
$$


For the stripper, the interfacial fugacity is determined during the solution of the enhancement factor.

As per the original publication, the log-mean between the top and bottom of the columns are used for the bulk $\mathrm{CO}_{2}$ fugacities.

\section{S5.16. Pump cost curves}

The pump cost curves used in the original publication did not have a flow rate range suitable for this work.

The updated cost curves for a general carbon steel centrifugal pump with a maximum outlet pressure of $1035 \mathrm{kPa}$, are now used from Timmerhaus et al..$^{24}$

$$
C= \begin{cases}-9998893 Q^{2}+318769.0 Q+1017.347, & Q \leq 0.009 \\ \exp [0.3848224 \cdot \ln (Q)+9.822074], & Q>0.009\end{cases}
$$

Where C is the cost of the pump in US\$ for a CEPCI of 390.4, and Q is the flow rate in $\mathrm{m}^{3} \cdot \mathrm{s}^{-1}$.

In cases where stainless steel is required, a material factor of 2.4 is applied for $304 \mathrm{SS}$ to the carbon steel cost. 


\section{S6. References}

(1) Garðarsdóttir, S. Ó.; Normann, F.; Skagestad, R.; Johnsson, F., Investment costs and CO2 reduction potential of carbon capture from industrial plants - A Swedish case study. International Journal of Greenhouse Gas Control 2018, 76, 111-124, DOI: 10.1016/j.ijggc.2018.06.022.

(2) Ho, M. T.; Allinson, G. W.; Wiley, D. E., Comparison of MEA capture cost for low CO2 emissions sources in Australia. International Journal of Greenhouse Gas Control 2011, 5 (1), 49-60, DOI: 10.1016/j.ijggc.2010.06.004.

(3) Mores, P. L.; Manassaldi, J. I.; Scenna, N. J.; Caballero, J. A.; Mussati, M. C.; Mussati, S. F., Optimization of the design, operating conditions, and coupling configuration of combined cycle power plants and CO2 capture processes by minimizing the mitigation cost. Chemical Engineering Journal 2018, 331, 870-894, DOI: 10.1016/j.cej.2017.08.111.

(4) Nuchitprasittichai, A.; Cremaschi, S., Sensitivity of amine-based CO2 capture cost: The influences of $\mathrm{CO} 2$ concentration in flue gas and utility cost fluctuations. International Journal of Greenhouse Gas Control 2013, 13, 34-43, DOI: 10.1016/j.ijggc.2012.12.012.

(5) Feron, P.; Cousins, A.; Jiang, K.; Zhai, R.; Shwe Hla, S.; Thiruvenkatachari, R.; Burnard, K., Towards Zero Emissions from Fossil Fuel Power Stations. International Journal of Greenhouse Gas Control 2019, 87, 188-202, DOI: 10.1016/j.ijggc.2019.05.018.

(6) Andersson, V.; Franck, P.-y.; Berntsson, T., Techno-economic analysis of excess heat driven postcombustion CCS at an oil refinery. International Journal of Greenhouse Gas Control 2016, 45, 130-138, DOI: $10.1016 /$ j.ijggc.2015.12.019.

(7) Abu-Zahra, M. R. M.; Niederer, J. P. M.; Feron, P. H. M.; Versteeg, G. F., CO2 capture from power plants: Part II. A parametric study of the economical performance based on monoethanolamine. International Journal of Greenhouse Gas Control 2007, 1 (2), 135-142, DOI: 10.1016/s17505836(07)00032-1.

(8) Agbonghae, E. O.; Hughes, K. J.; Ingham, D. B.; Ma, L.; Pourkashanian, M., Optimal Process Design of Commercial-Scale Amine-Based CO2 Capture Plants. Industrial \& Engineering Chemistry Research 2014, 53 (38), 14815-14829, DOI: 10.1021/ie5023767.

(9) Hasan, M. M. F.; Baliban, R. C.; Elia, J. A.; Floudas, C. A., Modeling, Simulation, and Optimization of Postcombustion CO2 Capture for Variable Feed Concentration and Flow Rate. 1. Chemical Absorption and Membrane Processes. Industrial \& Engineering Chemistry Research 2012, 51 (48), 1564215664, DOI: 10.1021/ie301571d.

(10) Bui, M.; Flø, N. E.; de Cazenove, T.; Mac Dowell, N., Demonstrating flexible operation of the Technology Centre Mongstad (TCM) CO2 capture plant. International Journal of Greenhouse Gas Control 2020, 93, DOI: 10.1016/j.ijggc.2019.102879.

(11) Faramarzi, L.; Thimsen, D.; Hume, S.; Maxon, A.; Watson, G.; Pedersen, S.; Gjernes, E.; Fostås, B. F.; Lombardo, G.; Cents, T.; Morken, A. K.; Shah, M. I.; de Cazenove, T.; Hamborg, E. S., Results from MEA Testing at the CO2 Technology Centre Mongstad: Verification of Baseline Results in 2015. Energy Procedia 2017, 114, 1128-1145, DOI: 10.1016/j.egypro.2017.03.1271.

(12) Alhajaj, A.; Mac Dowell, N.; Shah, N., A techno-economic analysis of post-combustion CO 2 capture and compression applied to a combined cycle gas turbine: Part II. Identifying the costoptimal control and design variables. International Journal of Greenhouse Gas Control 2016, 52, 331-343, DOI: $10.1016 /$ j.ijggc.2016.07.008.

(13) Canepa, R.; Wang, M., Techno-economic analysis of a CO2 capture plant integrated with a commercial scale combined cycle gas turbine (CCGT) power plant. Applied Thermal Engineering 2015, 74, 10-19, DOI: 10.1016/j.applthermaleng.2014.01.014.

(14) Jiang, K.; Feron, P.; Cousins, A.; Zhai, R.; Li, K., Achieving Zero/Negative-Emissions Coal-Fired Power Plants Using Amine-Based Postcombustion CO2 Capture Technology and Biomass 
Cocombustion. Environmental Science \& Technology 2020, 54 (4), 2429-2438, DOI: 10.1021 /acs.est.9b07388.

(15) Wilson, Z. T.; Sahinidis, N. V., The ALAMO approach to machine learning. Computers \& Chemical Engineering 2017, 106, 785-795, DOI: 10.1016/j.compchemeng.2017.02.010.

(16) Rocha, J. A.; Bravo, J. L.; Fair, J. R., Distillation columns containing structured packings: a comprehensive model for their performance. 1. Hydraulic models. Industrial \& Engineering Chemistry Research 1993, 32 (4), 641-651, DOI: 10.1021/ie00016a010.

(17) Gabrielsen, J.; Michelsen, M. L.; Stenby, E. H.; Kontogeorgis, G. M., A Model for Estimating CO2 Solubility in Aqueous Alkanolamines. Industrial \& Engineering Chemistry Research 2005, 44 (9), 33483354, DOI: $10.1021 /$ ie048857i.

(18) Park, S. H.; Lee, K. B.; Hyun, J. C.; Kim, S. H., Correlation and Prediction of the Solubility of Carbon Dioxide in Aqueous Alkanolamine and Mixed Alkanolamine Solutions. Industrial \& Engineering Chemistry Research 2002, 41 (6), 1658-1665, DOI: 10.1021/ie010252o.

(19) Peng, D.-Y.; Robinson, D. B., A New Two-Constant Equation of State. Industrial \& Engineering Chemistry Fundamentals 1976, 15 (1), 59-64, DOI: 10.1021/i160057a011.

(20) Qian, J.-W.; Privat, R.; Jaubert, J.-N., Predicting the Phase Equilibria, Critical Phenomena, and Mixing Enthalpies of Binary Aqueous Systems Containing Alkanes, Cycloalkanes, Aromatics, Alkenes, and Gases (N2, CO2, H2S, H2) with the PPR78 Equation of State. Industrial \& Engineering Chemistry Research 2013, 52 (46), 16457-16490, DOI: 10.1021/ie402541h.

(21) Poling, B.; Prausnitz, J.; O'Connell, J., The Properties of Gases and Liquids. 5th ed.; McGraw-Hill: New York, 2001.

(22) Burkholder, J. B.; Sander, S. P.; Abbatt, J.; Barker, J. R.; Huie, R. E.; Kolb, C. E.; Kurylo, M. J.; Orkin, V. L.; Wilmouth, D. M.; Wine, P. H., Chemical Kinetics and Photochemical Data for Use in Atmospheric Studies, Evaluation No. 18. Jet Propulsion Laboratory: Pasadena, 2015.

(23) Jou, F.-Y.; Mather, A. E.; Otto, F. D., The solubility of CO2 in a 30 mass percent monoethanolamine solution. The Canadian Journal of Chemical Engineering 1995, 73 (1), 140-147, DOI: 10.1002/cjce.5450730116.

(24) Peters, M. S.; Timmerhaus, K. D.; West, R. E., Plant Design and Economics for Chemical Engineers. 5th ed.; McGraw-Hill: Boston, 2006.

(25) Amundsen, T. G.; Øi, L. E.; Eimer, D. A., Density and Viscosity of Monoethanolamine + Water + Carbon Dioxide from $(25 \text { to } 80)^{\circ} \mathrm{C}$. Joumal of Chemical \& Engineering Data 2009, 54 (11), 3096-3100, DOI: $10.1021 /$ je $900188 \mathrm{~m}$.

(26) Ko, J.-J.; Tsai, T.-C.; Lin, C.-Y.; Wang, H.-M.; Li, M.-H., Diffusivity of Nitrous Oxide in Aqueous Alkanolamine Solutions. Journal of Chemical \& Engineering Data 2001, 46 (1), 160-165, DOI: 10.1021/je000138x.

(27) Gilliland, E. R., Diffusion Coefficients in Gaseous Systems. Industrial \& Engineering Chemistry 1934, 26 (6), 681-685, DOI: 10.1021/ie50294a020.

(28) Fairbanks, D. F.; Wilke, C. R., Diffusion Coefficients in Multicomponent Gas Mixtures. Industrial \& Engineering Chemistry 1950, 42 (3), 471-475, DOI: 10.1021/ie50483a022.

(29) Rocha, J. A.; Bravo, J. L.; Fair, J. R., Distillation Columns Containing Structured Packings: A Comprehensive Model for Their Performance. 2. Mass-Transfer Model. Industrial \& Engineering Chemistry Research 1996, 35 (5), 1660-1667, DOI: 10.1021/ie940406i.

(30) Harun, N.; Nittaya, T.; Douglas, P. L.; Croiset, E.; Ricardez-Sandoval, L. A., Dynamic simulation of MEA absorption process for CO2 capture from power plants. International Journal of Greenhouse Gas Control 2012, 10, 295-309, DOI: 10.1016/j.ijggc.2012.06.017.

(31) Hoff, K. A.; Juliussen, O.; Falk-Pedersen, O.; Svendsen, H. F., Modeling and Experimental Study of Carbon Dioxide Absorption in Aqueous Alkanolamine Solutions Using a Membrane Contactor. Industrial \& Engineering Chemistry Research 2004, 43 (16), 4908-4921, DOI: 10.1021/ie034325a. 
(32) Snijder, E. D.; te Riele, M. J. M.; Versteeg, G. F.; van Swaaij, W. P. M., Diffusion coefficients of several aqueous alkanolamine solutions. Journal of Chemical \& Engineering Data 1993, 38 (3), 475-480, DOI: $10.1021 / \mathrm{je} 00011 \mathrm{a} 037$.

(33) Shahid, M. Z.; Maulud, A. S.; Bustam, M. A.; Suleman, H.; Halim, H. N. A.; Shariff, A. M., RateBased Modeling for Packed Absorption Column of the MEA-CO2-Water System at High-Pressure and High-CO2 Loading Conditions. Industrial \& Engineering Chemistry Research 2019, 58 (27), 1223512246, DOI: 10.1021/acs.iecr.9b01482. 